\title{
Dual formulations in critical state problems
}

\author{
JOHN W. BARRETT ${ }^{\dagger}$ \\ Department of Mathematics, Imperial College, London SW7 2AZ, UK \\ AND \\ LEONID PRIGOZHIN \\ Department of Solar Energy and Environmental Physics, \\ J. Blaustein Institute for Desert Research, \\ Ben-Gurion University of the Negev, Sede Boqer Campus, 84990 Israel
}

[Received 27 September 2005]

\begin{abstract}
Similar evolutionary variational inequalities appear as convenient formulations for continuous models for sandpile growth, magnetization of type-II superconductors, and evolution of some other dissipative systems characterized by the multiplicity of metastable states, long-range interactions, avalanches, and hysteresis. The origin of this similarity is that these are quasistationary models in which the multiplicity of metastable states is a consequence of a unilateral condition of equilibrium (critical-state constraint). Existing variational formulations for critical-state models of sandpiles and superconductors are convenient for modeling only the "primary" variables (evolving pile shape and magnetic field, respectively). The conjugate variables (the surface sand flux and the electric field) are also of interest in various applications. Here we derive dual variational formulations, which have some similarities to mixed variational inequalities in plasticity, for the sandpile and superconductor models. We then approximate them by fully practical finite element methods based on the lowest order Raviart-Thomas element. We prove convergence of these approximations, and hence existence of a solution, to these dual formulations. Finally, we present some numerical experiments.
\end{abstract}

2000 Mathematics Subject Classification: 35D05, 35K85, 49J40, 49M29, 65M12, 65M60, 82C27.

Keywords: Variational inequalities; critical-state problems; duality; finite elements; existence; convergence analysis.

\section{Introduction}

Sandpiles and type-II superconductors are examples of spatially extended open dissipative systems which have infinitely many metastable states; but, driven by the external forces, tend to organize themselves into a marginally stable "critical state" and are then able to demonstrate almost instantaneous long-range interactions. The evolution of such systems is often accompanied by sudden collapses, like sandpile avalanches, and hysteresis. Although these are dissipative systems of a different nature, their continuous models are equivalent to similar variational (or quasivariational) inequalities (see [14] and the references therein). The origin of this similarity is that these models are quasistationary models of equilibrium and the multiplicity of metastable states is a consequence of a unilateral equilibrium condition. The rate with which such a system adjusts itself to the changing

\footnotetext{
†E-mail: jwb@ic.ac.uk

‡E-mail: leonid@cs.bgu.ac.il
} 
external conditions is determined implicitly and appears in the model as a Lagrange multiplier. Typically, the multiplier is eliminated in transition to a variational formulation written in terms of a "primary" variable (surface of a sandpile, magnetic field in a superconductor, stress tensor in elastoplasticity, etc.) In many situations, however, the Lagrange multiplier or, equivalently, a "dual" variable (sand flux upon the pile surface, electric field, and strain tensor, respectively) also has to be found. We present, for both the sandpile and the superconductivity problem, the variational formulations written for the dual variables. On discretization these formulations yield an efficient algorithm to compute the dual and primal variables simultaneously. Only the simplest version of each problem is considered.

We remark that these dual formulations have some similarities to mixed variational inequalities in elastoplasticity [10]. We note also that the sandpile model is strongly related to the MongeKantorovich problem of optimal mass transportation with linear cost, and our algorithm can be used (see [15]) for the numerical approximation of this classical problem too.

\section{Sandpiles}

Let sand be poured out onto a rigid support surface, $y=w^{0}(\underline{x})$, given in a bounded open subset $\Omega$ of $\mathbb{R}^{2}$ with a Lipschitz boundary $\partial \Omega$. If the support boundary is open, a model for pile surface evolution can be written as

$$
\partial_{t} w+\underline{\nabla} \cdot \underline{q}=f,\left.\quad w\right|_{t=0}=w^{0},\left.\quad w\right|_{\partial \Omega}=\left.w^{0}\right|_{\partial \Omega},
$$

where $w(\underline{x}, t)$ is the unknown pile surface, $f(\underline{x}, t) \geqslant 0$ is the given source density, $\underline{q}(\underline{x}, t)$ is the unknown horizontal projection of the flux of sand pouring down the pile surface. If the support has no slopes steeper than the sand angle of repose, $k=\tan \alpha$, and $\left|\underline{\nabla} w^{0}\right| \leqslant k$, the simplest constitutive relations for this model read: (i) the flux $q$ is directed towards the steepest decent of the surface, (ii) the surface slope cannot exceed the critical angle $\alpha$, and (iii) the flux is zero upon subcritical slopes. Equivalently, one can write $q=-m \underline{\nabla} w$ and show that $m(\underline{x}, t) \geqslant 0$ is the Lagrange multiplier related to the constraint $|\underline{\nabla} w| \leqslant k$ and satisfies $m\left(|\underline{\nabla} w|^{2}-k^{2}\right)=0$. The above model, which we denote by $(\mathrm{E})$, can be reformulated as a variational inequality of the first kind for $w$ :

(P) Find $w \in L^{\infty}\left(0, T ; K_{w^{0}}(k)\right) \cap H^{1}\left(0, T ; L^{2}(\Omega)\right)$

$$
\int_{0}^{T}\left(\partial_{t} w-f, \eta-w\right) \mathrm{d} t \geqslant 0 \quad \forall \eta \in L^{\infty}\left(0, T ; K_{w^{0}}(k)\right),
$$

where $K_{w^{0}}(k):=\left\{\eta \in W^{1, \infty}(\Omega):|\underline{\nabla} \eta| \leqslant k\right.$ a.e. and $\left.\left.\eta\right|_{\partial \Omega}=\left.w^{0}\right|_{\partial \Omega}\right\}$ and $(\cdot, \cdot)$ is the standard $L^{2}(\Omega)$ inner product.

If $f \in L^{2}\left(\Omega_{T}\right)$, where $\Omega_{T}:=\Omega \times(0, T)$, and $w^{0} \in K_{w^{0}}(k)$, then existence and uniqueness of a solution to $(\mathrm{P})$ is proved in [13]. Moreover, it is shown there that $(\mathrm{P})$ is equivalent to the following weak formulation of $(E)$ :

$\left(\mathrm{E}_{1}\right)$ Find $w \in L^{\infty}\left(0, T ; K_{w^{0}}(k)\right) \cap H^{1}\left(0, T ; L^{2}(\Omega)\right)$ and $m \in\left[L^{\infty}\left(\Omega_{T}\right)\right]^{*}$ such that $w(0)=w^{0}$ and

$$
\begin{aligned}
\int_{0}^{T}\left(\partial_{t} w-f, \eta\right) \mathrm{d} t+\langle m, \underline{\nabla} w \cdot \underline{\nabla} \eta\rangle_{L^{\infty}\left(\Omega_{T}\right)}=0 & \forall \eta \in L^{\infty}\left(0, T ; W_{0}^{1, \infty}(\Omega)\right), \\
\langle m, \phi\rangle_{L^{\infty}\left(\Omega_{T}\right)} \geqslant 0 & \forall \phi \in L^{\infty}\left(\Omega_{T}\right) \text { with } \phi \geqslant 0, \\
\left\langle m,|\underline{\nabla} w|^{2}-k^{2}\right\rangle_{L^{\infty}\left(\Omega_{T}\right)}=0 . &
\end{aligned}
$$


Here $[\cdot]^{*}$ denotes the corresponding dual space and $\langle\cdot, \cdot\rangle_{V}$ the duality pairing on $[V]^{*} \times V$. We note that the same model of sand surface evolution was independently derived and studied in [1].

Simple analytical solutions of the inequality problem $(\mathrm{P})$ describe piles generated on open supports with $w^{0} \equiv 0$. For the point source $f=a \delta\left(\underline{x}-\underline{x}_{0}\right)$, a conical pile with critical slopes grows until its base touches the domain boundary $\partial \Omega$; the surface flux can also be easily calculated [12]. Then there appears a runway connecting the cone apex with the boundary and the pile growth stops: all additional sand just follows the runway and leaves the system. On the other hand, if $f>0$ everywhere in $\Omega$, the final stationary shape of the pile is different: $w(\underline{x})=k \operatorname{dist}(\underline{x}, \partial \Omega)$.

For $f \geqslant 0$, the general stationary solution and an integral representation formula for the corresponding Lagrange multiplier $m$, determining the surface sand flux $q$, have also been obtained recently in [6]. It is, however, not easy to compute the Lagrange multiplier using this formula. In the non-stationary case, determining the surface flux $q$ remains difficult even if the unique solution $w$ to $[1.2$ is found. To compute both these variables, we now derive a dual variational formulation of the evolutionary problem. Below, for later developments (sand problems with obstacles and superconductivity problems), we allow $k$ to be possibly piecewise constant in space; that is,

$$
k(x)=k^{(i)} \in \mathbb{R}^{+} \quad \forall x \in \Omega^{(i)}, i=1 \rightarrow I,
$$

where $\Omega^{(i)}$ are disjoint open subsets of $\Omega$ with Lipschitz boundaries and $\bar{\Omega} \equiv \bigcup_{i=1}^{I} \overline{\Omega^{(i)}}$.

Let $\{w, q\}$ satisfy the relations (i)-(iii) in (E) above. Then, for any test field $\underline{v}$,

$$
\underline{\nabla} w \cdot(\underline{v}-\underline{q}) \geqslant-|\underline{\nabla} w||\underline{v}|-\underline{\nabla} w \cdot \underline{q}=-|\underline{\nabla} w||\underline{v}|+k|\underline{q}| \geqslant-k|\underline{v}|+k|\underline{q}| .
$$

Hence we have

$$
(\underline{\nabla} w, \underline{v}-\underline{q}) \geqslant \sum_{i=1}^{I} k^{(i)} \int_{\Omega^{(i)}}|\underline{q}|-\sum_{i=1}^{I} k^{(i)} \int_{\Omega^{(i)}}|\underline{v}| .
$$

Since

$$
(\underline{\nabla} w, \underline{v}-\underline{q})=-(w, \underline{\nabla} \cdot(\underline{v}-\underline{q}))+\oint_{\partial \Omega} w^{0}\left(v_{n}-q_{n}\right),
$$

where $v_{n}$ is the normal component of $\underline{v}$ on $\partial \Omega$, we have

$$
\sum_{i=1}^{I} k^{(i)} \int_{\Omega^{(i)}}|\underline{v}|-\sum_{i=1}^{I} k^{(i)} \int_{\Omega^{(i)}}|\underline{q}|-(w, \underline{\nabla} \cdot(\underline{v}-\underline{q}))+\oint_{\partial \Omega} w^{0}\left(v_{n}-q_{n}\right) \geqslant 0 .
$$

Let $\Omega_{T}^{(i)}:=\Omega^{(i)} \times(0, T)$ and $\underline{\Phi}$ be the Banach space of vector functions $\phi: \bigcup_{i=1}^{I} \Omega_{T}^{(i)} \rightarrow \mathbb{R}^{2}$ such that for each $i$ the restriction $\left.\underline{\phi}\right|_{\Omega_{T}^{(i)}}$ is continuous and can be extended to a function from $\left[C\left(\overline{\Omega_{T}^{(i)}}\right)\right]^{2}$. The elements of the dual space $\underline{\mathcal{M}}:=[\underline{\Phi}]^{*}$ can be represented as $\underline{v}=\left(\underline{v}^{(1)}, \ldots, \underline{v}^{(I)}\right)$, where $\underline{v}^{(i)} \in \underline{\mathcal{M}}\left(\Omega_{T}^{(i)}\right):=\left[\left[C\left(\overline{\Omega_{T}^{(i)}}\right)\right]^{2}\right]^{*}$ is a vector Radon measure, $\|\underline{v}\|_{\underline{\mathcal{M}}}:=\sum_{i=1}^{I}\left\|\underline{v}^{(i)}\right\|_{\mathcal{M}\left(\Omega_{T}^{(i)}\right)}$, and $\langle\underline{\phi}, \underline{v}\rangle_{\underline{\Phi}}:=\sum_{i=1}^{I}\left\langle\left.\underline{\phi}\right|_{\Omega_{T}^{(i)}}, \underline{v}^{(i)}\right\rangle_{\left[C\left(\Omega_{T}^{(i)}\right)\right]^{2}}$. To simplify our notation, below we will write

$$
\langle k,|\underline{v}|\rangle:=\sum_{i=1}^{I}\left\langle k^{(i)},\left|\underline{v}^{(i)}\right|\right\rangle \frac{}{C\left(\overline{\Omega_{T}^{(i)}}\right.} .
$$


Noting from 1.1 that

$$
w(t)=w^{0}+\int_{0}^{t} f(r) \mathrm{d} r-\underline{\nabla} \cdot\left(\int_{0}^{t} \underline{q}(r) \mathrm{d} r\right)
$$

and using (1.8), we finally obtain our dual formulation of $(\mathrm{P})$, the following variational inequality of the second kind for $\underline{q}$ :

(Q) Find $\underline{q} \in \underline{V} \mathcal{M}(\operatorname{div})$ such that

$$
\int_{0}^{T}\left[\left(\underline{\nabla} \cdot\left(\int_{0}^{t} \underline{q}(r) \mathrm{d} r\right), \underline{\nabla} \cdot(\underline{v}-\underline{q})\right)+\mathcal{F}(\underline{v}-\underline{q})\right] \mathrm{d} t+\langle k,|\underline{v}|\rangle-\langle k,|\underline{q}|\rangle \geqslant 0
$$

for all $\underline{v} \in \widehat{V}_{\mathcal{M}}(\operatorname{div})$

Here $\mathcal{F}(\underline{v}):=\oint_{\partial \Omega} w^{0} v_{n}-\left(w^{0}+\int_{0}^{t} f(r) \mathrm{d} r, \underline{\nabla} \cdot \underline{v}\right)$ and

$$
\underline{V}_{\mathcal{M}}(\operatorname{div}):=\left\{\underline{v} \in \underline{\mathcal{M}}: \operatorname{div} \underline{v} \in L^{2}\left(\Omega_{T}\right)\right\} .
$$

In addition, the test space is defined as

$$
\begin{gathered}
\widehat{V}_{\mathcal{M}}\left(\text { div) }:=\text { the strong closure of }\left[C^{\infty}\left(\bar{\Omega}_{T}\right)\right]^{2}\right. \text { with respect to the norm } \\
\qquad\|\underline{v}\|_{\underline{\mathcal{M}}(\mathrm{div})}:=\|\underline{v}\|_{\mathcal{M}}+\|\underline{\nabla} \cdot \underline{v}\|_{L^{2}\left(\Omega_{T}\right)} .
\end{gathered}
$$

As $\left[C^{\infty}\left(\bar{\Omega}_{T}\right)\right]^{2}$ is dense in $\underline{V}_{2}(\operatorname{div}):=\left\{\underline{v} \in\left[L^{2}\left(\Omega_{T}\right)\right]^{2}: \underline{\nabla} \cdot \underline{v} \in L^{2}\left(\Omega_{T}\right)\right\}$ (recall [17, p. 13]), we clearly have $\underline{V}_{2}$ (div) $\subseteq \widehat{V}_{\mathcal{M}}$ (div) $\subseteq \underline{V}_{\mathcal{M}}($ div). This choice of test space is discussed in more detail at the end of this section.

Given $f \in L^{2}\left(\Omega_{T}\right)$ and $w^{0} \in K_{0}(k)$, we will prove (see Theorem 3.1 below) the existence of a solution to (Q). In addition, we will show (see Theorem 3.2 below) that the corresponding $w$, defined via 1.9$)$, is such that $w \in L^{\infty}\left(0, T ; K_{0}(k)\right)$ and solves the primal variational inequality (P), with $K_{w^{0}}(k)$ replaced by $K_{0}(k)$, and is therefore unique. Moreover, we will show that (Q) is equivalent to the following weak formulation of $(\mathrm{E})$ :

$\left(\mathrm{E}_{2}\right)$ Find $w \in L^{\infty}\left(0, T ; K_{0}(k)\right) \cap H^{1}\left(0, T ; L^{2}(\Omega)\right)$ and $q \in \underline{V} \mathcal{M}(\operatorname{div})$ such that $w(0)=w^{0}$ and

$$
\int_{0}^{T}\left(\partial_{t} w+\underline{\nabla} \cdot \underline{q}-f, \eta\right) \mathrm{d} t=0 \quad \forall \eta \in L^{2}\left(\Omega_{T}\right),
$$

with

$$
\int_{0}^{T}(w, \underline{\nabla} \cdot \underline{q}) \mathrm{d} t=\langle k,|\underline{q}|\rangle .
$$

We note that $1.13 \mathrm{~b}$ is another (weaker) formulation of the constitutive relation. Indeed, if $\underline{q}$ is regular enough (belongs to $\left.\left[\left[L^{\infty}\left(\Omega_{T}\right)\right]^{2}\right]^{*}\right)$ then $\left.1.13 b\right)$ and $w \in L^{\infty}\left(0, T ; K_{0}(k)\right)$ imply that $\underline{q}=-m \underline{\nabla} w$, where $m \in\left[L^{\infty}\left(\Omega_{T}\right)\right]^{*}$ and satisfies $\left.1.3 \mathrm{~b}-1.3 \mathrm{c}\right)$.

\section{Superconductors}

Phenomenologically, the magnetic field penetration into type-II superconductors can be understood as a nonlinear eddy current problem. Let a long cylindrical superconductor with a simply connected cross-section $\Omega$ be placed into a non-stationary uniform external magnetic field $\underline{h}_{e}(t)$ parallel to the 
cylindrical generators. According to Faraday's law, time variations of this field induce in a conductor an electric field $\underline{e}$ leading to a current $j$ parallel to the cross-section plane; this current induces a magnetic field $\underline{h}(\underline{x}, t)$ parallel to $\underline{h}_{e}$. Omitting the displacement current in Maxwell's equations and scaling the magnetic permeability to be unity, we obtain the following model:

$$
\partial_{t}\left(h+h_{e}\right)+\operatorname{curl} \underline{e}=0, \quad \underline{\operatorname{curl}} h=\underline{j},\left.\quad h\right|_{t=0}=h^{0}(\underline{x}),\left.\quad h\right|_{\partial \Omega}=0,
$$

where curl $\underline{u}=\partial_{x_{1}} u_{2}-\partial_{x_{2}} u_{1}$ and $\underline{\operatorname{curl}} u=\left(\partial_{x_{2}} u,-\partial_{x_{1}} u\right)^{T}$. Instead of the usual Ohm law, a multivalued current-voltage relation (the Bean model) is often employed for type-II superconductors. It is postulated that (i) the electric field $\underline{e}$ and the current density $j$ have the same direction, (ii) the current density $j(\underline{x}, t)$ cannot exceed some critical value, $j_{c}(\underline{x})$, and (iii) if the current is subcritical, the electric field is zero. One can write $\underline{e}=\rho j$ and show that the effective resistivity, $\rho(\underline{x}, t) \geqslant 0$, is the Lagrange multiplier related to current density constraint $|j(\underline{x}, t)| \leqslant j_{c}(\underline{x})$. Using conditions (i)-(iii) we can eliminate the electric field from the model. This yields, for given $h^{0} \in K_{0}\left(j_{c}\right)$ and $h_{e} \in H^{1}(0, T, \mathbb{R})$, the variational inequality of the first kind for $h$ :

- Find $h \in L^{\infty}\left(0, T ; K_{0}\left(j_{c}\right)\right) \cap H^{1}\left(0, T ; L^{2}(\Omega)\right)$ such that $h(0)=h^{0}$ and

$$
\int_{0}^{T}\left(\partial_{t}\left(h+h_{e}\right), \eta-h\right) \mathrm{d} t \geqslant 0 \quad \forall \eta \in L^{\infty}\left(0, T ; K_{0}\left(j_{c}\right)\right) .
$$

This inequality for $h$ can be approximated numerically and even solved analytically in some cases.

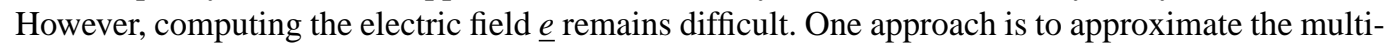
valued Bean current-voltage relation by the power law, $|\underline{e}|=e_{0}\left(|\underline{\operatorname{curl}} h| / j_{c}\right)^{p}$ with $p \gg 1$ (see [5, 4]). While such an approach can be efficient for computing the primal variable, $h$, the calculation of $\underline{e}$ using this formula may be unstable and smoothing of $h$ is needed if $p$ is large; see [5] for the details. Recently, a numerical algorithm based on optimal control theory, requiring the integration along paths of the magnetic flux penetration, has been proposed in [2]. Here we consider a dual variational formulation which can be derived similarly to that for the sandpile model. The dual formulation can be employed to find both fields simultaneously, the algorithm is stable, even if the electric field is singular, and requires neither the determination of the penetration paths nor the integration along them. In addition, the dual formulation can be extended to general threedimensional configurations [14].

For cylindrical superconductors and on assuming, similarly to (1.4), that $j_{c}$ is piecewise constant, this formulation reads:

- Find $\underline{e} \in \underline{V} \mathcal{M}(\operatorname{curl}):=\left\{\underline{v} \in \underline{\mathcal{M}}: \operatorname{curl} \underline{v} \in L^{2}\left(\Omega_{T}\right)\right\}$ such that

$$
\begin{array}{r}
\int_{0}^{T}\left[\left(\operatorname{curl}\left(\int_{0}^{t} \underline{e}(r) \mathrm{d} r\right), \operatorname{curl}(\underline{v}-\underline{e})\right)+\mathcal{F}(\underline{v}-\underline{e})\right] \mathrm{d} t+\left\langle j_{c},|\underline{v}|\right\rangle-\left\langle j_{c},|\underline{e}|\right\rangle \geqslant 0 \\
\forall \underline{v} \in \underline{\widehat{V}}_{\mathcal{M}}(\operatorname{curl}) .
\end{array}
$$

Here $\mathcal{F}(\underline{v})=\left(h_{e}(t)-h_{e}(0)-h^{0}, \operatorname{curl} \underline{v}\right)$ and $\widehat{\widehat{V}}_{\mathcal{M}}\left(\right.$ curl) is defined similarly to $\underline{\underline{V}}_{\mathcal{M}}$ (div) (recall (1.12), with div replaced by curl. The primary variable, $h$, is then found from the analogue of (1.9),

$$
h(t)=h^{0}+h_{e}(0)-h_{e}(t)-\operatorname{curl}\left(\int_{0}^{t} \underline{e}(r) \mathrm{d} r\right) .
$$


The simple transformation $R: \underline{e}=\left(e_{1}, e_{2}\right)^{T} \mapsto\left(e_{2},-e_{1}\right)^{T}$ maps $\underline{V}_{\mathcal{M}}\left(\right.$ div) to $\underline{V}_{\mathcal{M}}$ (curl), $\widehat{V}_{\mathcal{M}}$ (div) to $\widehat{\underline{V}}_{\mathcal{M}}$ (curl), and enables us to apply the same theory and numerical approximation for the dual sandpile model (Q), (1.10, to the dual superconductor model (1.16).

The outline of this paper is as follows. In the next section we introduce a fully discrete finite element approximation, $\left(\mathrm{Q}_{\varepsilon}^{h, \tau}\right)$, of the dual formulation, $(\mathrm{Q})$, of the sandpile model. It is based on smoothing the non-differentiable functional $\langle k,|\underline{v}|\rangle$ by introducing $|\underline{v}|_{\varepsilon}:=\left(|\underline{v}|^{2}+\varepsilon^{2}\right)^{1 / 2}$, and employing the lowest order Raviart-Thomas element for the spatial discretization with vertex sampling on the non-linear term. We then establish stability bounds on this approximation, independent of the mesh parameters, $h$ and $\tau$, and the regularization parameter, $\varepsilon$. In Section 3 we prove subsequence convergence of this approximation, and hence existence of a solution to (Q). As stated above all these results for the dual formulation, $(\mathrm{Q})$, of the sandpile model carry over to the corresponding superconductor model via the transformation $R$. Finally, in Section 4 we present some numerical experiments based on the discretization $\left(\mathrm{Q}_{\varepsilon}^{h, \tau}\right)$ for both the sandpile and superconductor models.

We end this section with a few remarks about the notation employed in this paper. Above and throughout we adopt the standard notation for Sobolev spaces, denoting the norm of $W^{\ell, r}(D)$ $(\ell \in \mathbb{N}, r \in[1, \infty])$ by $\|\cdot\|_{\ell, r, D}$ and the seminorm by $|\cdot|_{\ell, r, D}$. Of course, $|\cdot|_{0, r, D} \equiv\|\cdot\|_{0, r, D}$. We extend these norms and seminorms in the natural way to the corresponding spaces of vector-valued functions. For $r=2, W^{\ell, 2}(D)$ will be denoted by $H^{\ell}(D)$ with the associated norm and seminorm written as, respectively, $\|\cdot\|_{\ell, D}$ and $|\cdot|_{\ell, D}$. The measure of $D$ will be denoted by $|D|$.

Let $C(\bar{D})$ denote the space of continuous functions on $\bar{D}$. As one can identify $L^{1}(D)$ as a closed subspace of $\mathcal{M}(D)$, it is convenient to adopt the notation

$$
\int_{D}|\mu| \equiv\|\mu\|_{\mathcal{M}(D)}:=\sup _{\eta \in C(\bar{D})} \frac{\left|\langle\mu, \eta\rangle_{C(\bar{D})}\right|}{|\eta|_{0, \infty}, D}<\infty .
$$

We note that if $\left\{\mu_{j}\right\}_{j} \geqslant 0$ is a bounded sequence in $\mathcal{M}(D)$, then there exist a subsequence $\left\{\mu_{j_{\ell}}\right\}_{j_{\ell}} \geqslant 0$ and a $\mu \in \mathcal{M}(D)$ such that as $j_{\ell} \rightarrow \infty$,

$$
\mu_{j_{\ell}} \rightarrow \mu \quad \text { weakly in } \mathcal{M}(D) \text {, i.e. }\left\langle\mu_{j_{\ell}}-\mu, \eta\right\rangle_{C(\bar{D})} \rightarrow 0 \quad \forall \eta \in C(\bar{D}) .
$$

In addition,

$$
\liminf _{j_{\ell} \rightarrow \infty} \int_{D}\left|\mu_{j_{\ell}}\right| \geqslant \int_{D}|\mu|
$$

see e.g. [7, p. 5] and [9, p. 223].

For our proof of the existence of a solution to (Q) (see Theorem 3.1 below), we require the following density result for our test space: any element $\underline{v}$ can be approximated by a sequence $\left\{\underline{v}_{j}\right\}_{j} \geqslant 0$, where $\underline{v}_{j} \equiv \sum_{i=1}^{I} \underline{v}_{j}^{(i)}$ with $\underline{v}_{j}^{(i)} \in\left[C^{\infty}\left(\bar{\Omega}_{T}\right)\right]^{2}, i=1 \rightarrow I$, such that

$$
\limsup _{j \rightarrow \infty} \int_{\Omega_{T}}\left|\underline{v}_{j}^{(i)}\right| \leqslant \int_{\Omega_{T}^{(i)}}\left|\underline{v}^{(i)}\right|, \quad i=1 \rightarrow I,
$$

and as $j \rightarrow \infty$,

$$
\begin{aligned}
\left\langle\underline{v}_{j}^{(i)}, \underline{\phi}_{\left[C\left(\bar{\Omega}_{T}\right)\right]^{2}}\right. & \rightarrow\left\langle\underline{v}^{(i)}, \underline{\phi} \underline{[C}_{\left[C\left(\bar{\Omega}_{T}^{(i)}\right)\right]^{2}}\right. & & \forall \underline{\phi} \in\left[C_{0}\left(\Omega_{T}\right)\right]^{2}, i=1 \rightarrow I, \\
\underline{\nabla} \cdot \underline{v}_{j} & \rightarrow \underline{\nabla} \cdot \underline{v} & & \text { weakly in } L^{2}\left(\Omega_{T}\right) .
\end{aligned}
$$


Such a result is non-trivial for the Banach space $\underline{V}_{\mathcal{M}}$ (div) as it is not of local type; that is, $\underline{v} \in$ $\underline{V}_{\mathcal{M}}(\operatorname{div})$ and $\phi \in C^{\infty}\left(\bar{\Omega}_{T}\right)$ does not imply that $\phi \underline{v} \in \underline{V}_{\mathcal{M}}(\mathrm{div})$ and so the use of cut-off functions, as in the proof of Proposition 1.3 in [17], is not possible. On extending any $v \in V_{\mathcal{M}}$ (div) by zero and applying mollifiers it is a simple matter to establish $\sqrt{1.21 \mathrm{a}} \mathrm{b})$; but then $L^{2}\left(\Omega_{T}\right)$ in $\sqrt{1.21 \mathrm{c}}$ has to be replaced by $L^{2}(\omega)$ for any open set $\omega$ such that $\bar{C} \subset \Omega_{T}$. Clearly, the results $(1.21 \mathrm{a}-\mathrm{c})$ are trivially true for the choice $\widehat{\underline{V}}_{\mathcal{M}}$ (div) in 1.12 . Of course one could weaken the closure in (1.12) and these results would remain true. However, unless one can show that the corresponding $\underline{V}_{\mathcal{M}}(\mathrm{div}) \equiv \underline{V}_{\mathcal{M}}(\mathrm{div})$, which we are unable to do, there is no real gain in such a choice.

Finally, throughout $C$ denotes a generic constant independent of the mesh parameters, $h$ and $\tau$, and the regularization parameter, $\varepsilon$.

\section{Numerical approximation of $(Q)$}

In order to avoid the difficulty of traces on $\underline{V}_{\mathcal{M}}$ (div) (recall (1.10p), we will restrict the discussion in this paper to $\left.w^{0}\right|_{\partial \Omega}=0$. In addition, the results in this paper could, in principle, be extended to piecewise smooth $k$, but once again for ease of exposition we will restrict the discussion here to piecewise constant $k$.

Firstly, we gather together our assumptions on the data.

(A1) $\Omega \subset \mathbb{R}^{2}$ with a Lipschitz boundary $\partial \Omega, k$ satisfying $1.4, w^{0} \in K_{0}(k)$ and $f \in L^{2}\left(\Omega_{T}\right)$.

We then introduce $\bar{f}:[0, T] \rightarrow L^{2}(\Omega)$, where $\bar{f}(t)=w^{0}+\int_{0}^{t} f(r) \mathrm{d} r$. Then our dual formulation, 1.10, of (P) becomes:

(Q) Find $\underline{q} \in \underline{V}_{\mathcal{M}}(\mathrm{div})$ such that

$$
\int_{0}^{T}\left(\underline{\nabla} \cdot\left(\int_{0}^{t} \underline{q}(r) \mathrm{d} r\right)-\bar{f}, \underline{\nabla} \cdot(\underline{v}-\underline{q})\right) \mathrm{d} t+\langle k,|\underline{v}|\rangle-\langle k,|\underline{q}|\rangle \geqslant 0 \quad \forall \underline{v} \in \underline{\widehat{V}}_{\mathcal{M}}(\mathrm{div}) .
$$

The aim of this paper is to prove existence of, and approximate, solutions to (Q).

For any $\varepsilon \in \mathbb{R}^{+}$, we regularise the non-differentiable non-linearity $|\cdot|$ by introducing the strictly convex function $|\cdot|_{\varepsilon}: \mathbb{R}^{2} \rightarrow \mathbb{R}^{+}$defined by

$$
|\underline{a}|_{\varepsilon}:=\left(|\underline{a}|^{2}+\varepsilon^{2}\right)^{1 / 2}
$$

We note that for all $\underline{a}, \underline{b} \in \mathbb{R}^{2}$,

$$
\frac{\partial|\underline{a}|_{\varepsilon}}{\partial a_{i}}=\frac{a_{i}}{|\underline{a}|_{\varepsilon}}, \quad \frac{\partial^{2}|\underline{a}|_{\varepsilon}}{\partial a_{i} \partial a_{j}}=\frac{\delta_{i j}}{|\underline{a}|_{\varepsilon}}-\frac{a_{i} a_{j}}{|\underline{a}|_{\varepsilon}^{3}} \quad \text { and so } \quad \sum_{i, j=1}^{2} \frac{\partial^{2}|\underline{a}|_{\varepsilon}}{\partial a_{i} \partial a_{j}} b_{i} b_{j} \geqslant \frac{\varepsilon^{2}}{|\underline{a}|_{\varepsilon}^{3}}|\underline{b}|^{2} .
$$

Hence it follows that

$$
\frac{\underline{a}}{|\underline{a}|_{\varepsilon}} \cdot(\underline{a}-\underline{b}) \geqslant|\underline{a}|_{\varepsilon}-|\underline{b}|_{\varepsilon} .
$$

For ease of exposition, we assume that 
(A2) $\Omega$ and $\Omega^{(i)}, i=1 \rightarrow I$, are polygonal. Let $\left\{\mathcal{T}^{h}\right\}_{h>0}$ be a regular family of partitionings of $\Omega$ into disjoint open simplices $\sigma$ with $h_{\sigma}:=\operatorname{diam}(\sigma)$ and $h:=\max _{\sigma \in \mathcal{T}^{h}} h_{\sigma}$, so that $\bar{\Omega}=\bigcup_{\sigma \in \mathcal{T}^{h}} \bar{\sigma}$. In addition, for all $\sigma \in \mathcal{T}^{h}$, we have $\left.k\right|_{\sigma}=k_{\sigma} \in\left\{k^{(i)}\right\}_{i=1}^{I} ;$ and let $\mathcal{T}_{i}^{h} \subset \mathcal{T}^{h}$ be such that

$$
\overline{\Omega^{(i)}}=\bigcup_{\sigma \in \mathcal{T}_{i}^{h}} \bar{\sigma}, \quad i=1 \rightarrow I .
$$

We note that there is a clash of notation between $h$, the mesh length above, and the component of the magnetic field in 1.14. However, we want to keep the standard notation for these quantities; the meaning of $h$ will always be clear from the context.

Let $\underline{n}_{\sigma}$ be the outward unit normal to $\sigma$. We then introduce

$$
\begin{gathered}
\underline{V}^{h}:=\left\{\underline{v}^{h} \in\left[L^{\infty}(\Omega)\right]^{2}:\left.\underline{v}^{h}\right|_{\sigma}=\underline{a}_{\sigma}+b \underline{x}, \underline{a}_{\sigma} \in \mathbb{R}^{2}, b_{\sigma} \in \mathbb{R} \forall \sigma \in \mathcal{T}^{h}\right. \text { and } \\
\left.\underline{v}^{h} \cdot \underline{n}_{\sigma} \text { is continuous across simplex boundaries }\right\} \\
\subset\left\{\underline{v} \in\left[L^{\infty}(\Omega)\right]^{2}: \underline{\nabla} \cdot \underline{v} \in L^{2}(\Omega)\right\} \\
S^{h}:=\left\{\eta^{h} \in L^{\infty}(\Omega):\left.\eta^{h}\right|_{\sigma}=c_{\sigma} \in \mathbb{R} \forall \sigma \in \mathcal{T}^{h}\right\} .
\end{gathered}
$$

In order for our finite element approximation to be practical, we introduce $(\underline{v}, \underline{z})^{h}:=\sum_{\sigma \in \mathcal{T}^{h}}(\underline{v}, \underline{z})_{\sigma}^{h}$ with

$$
(\underline{v}, \underline{z})_{\sigma}^{h}:=\frac{1}{3}|\sigma| \sum_{j=1}^{3} \underline{v}\left(P_{j}^{\sigma}\right) \cdot \underline{z}\left(P_{j}^{\sigma}\right) \quad \forall \underline{v}, \underline{z} \in[C(\bar{\sigma})]^{2}, \forall \sigma \in \mathcal{T}^{h},
$$

where $\left\{P_{j}^{\sigma}\right\}_{j=1}^{3}$ are the vertices of $\sigma$. Hence $(\underline{v}, \underline{z})^{h}$ averages the integrand $\underline{v} \cdot \underline{z}$ over each simplex $\sigma$ at its vertices and hence is exact if $\underline{v} . \underline{z}$ is piecewise linear over the partitioning $\mathcal{T}^{h}$. We note for any $\underline{v}^{h} \in \underline{V}^{h}$ and any $\sigma \in \mathcal{T}^{h}$ that $\left|\underline{v}^{h}(x)\right| \leqslant \sum_{j=1}^{3}\left|\underline{v}^{h}\left(P_{j}^{\sigma}\right)\right| \lambda_{j}^{\sigma}(x)$ on $\bar{\sigma}$, where $\left\{\lambda_{j}^{\sigma}\right\}_{j=1}^{3}$ are the standard linear hat functions on $\sigma$ satisfying $\lambda_{j}^{\sigma}\left(P_{i}^{\sigma}\right)=\delta_{i j}, i, j=1 \rightarrow 3$; and so

$$
\int_{\sigma}\left|\underline{v}^{h}\right| \leqslant\left(\left|\underline{v}^{h}\right|, 1\right)_{\sigma}^{h}:=\frac{1}{3}|\sigma| \sum_{j=1}^{3}\left|\underline{v}^{h}\left(P_{j}^{\sigma}\right)\right|
$$

In addition, let $0=t_{0}<t_{1}<\cdots<t_{N-1}<t_{N}=T$ be a partitioning of $[0, T]$ into possibly variable time steps $\tau_{n}:=t_{n}-t_{n-1}, n=1 \rightarrow N$. We set $\tau:=\max _{n=1 \rightarrow N} \tau_{n}$. Our fully practical approximation of (Q) by $\underline{V}^{h}$, on employing 2.2 and 2.7 , is then:

$\left(\mathrm{Q}_{\varepsilon}^{h, \tau}\right)$ For $n \geqslant 1$, find $\underline{Q}_{\varepsilon}^{n} \in \underline{V}^{h}$ such that

$$
\tau_{n}\left(\underline{\nabla} \cdot \underline{Q}_{\varepsilon}^{n}, \underline{\nabla} \cdot \underline{v}^{h}\right)+\left(\frac{k \underline{Q}_{\varepsilon}^{n}}{\left|\underline{Q}_{\varepsilon}^{n}\right|_{\varepsilon}}, \underline{v}^{h}\right)^{h}=\left(G_{\varepsilon}^{n}, \underline{\nabla} \cdot \underline{v}^{h}\right) \quad \forall \underline{v}^{h} \in \underline{V}^{h},
$$

where $G_{\varepsilon}^{n}:=\bar{f}^{n}-\underline{\nabla} \cdot \underline{U}_{\varepsilon}^{n-1}, \bar{f}^{n}:=\bar{f}\left(t_{n}\right), n \geqslant 1$, and

$$
\underline{U}_{\varepsilon}^{n-1}:=\sum_{\ell=1}^{n-1} \tau_{\ell} \underline{Q}_{\varepsilon}^{\ell}, \quad n \geqslant 2, \quad \text { and } \quad \underline{U}_{\varepsilon}^{0}:=\underline{0} .
$$


One can then recover an approximation to $w\left(t_{n}\right), n=1 \rightarrow N$, by finding $W_{\varepsilon}^{n} \in S^{h}$ such that

$$
\left(W_{\varepsilon}^{n}, \eta^{h}\right)=\left(\bar{f}^{n}-\underline{\nabla} \cdot \underline{U}_{\varepsilon}^{n}, \eta^{h}\right) \quad \forall \eta^{h} \in S^{h} .
$$

LEMmA 2.1 Let the assumptions (A1) and (A2) hold. Then given $G_{\varepsilon}^{n}$, for all $\varepsilon>0$, for all regular partitionings $\mathcal{T}^{h}$ of $\Omega$, and for all $\tau_{n}>0$ there exists a unique solution $\underline{Q}_{\varepsilon}^{n} \in \underline{V}^{h}$, and $W_{\varepsilon}^{n} \in S^{h}$, to the $n^{\text {th }}$ step of $\left(\mathrm{Q}_{\varepsilon}^{h, \tau}\right)$.

Proof. On noting (2.3), we see that $2.9 \mathrm{a}$ ) is the Euler-Lagrange equation for the strictly convex minimization problem

$$
\min _{\underline{v}^{h} \in \underline{V}^{h}}\left\{\frac{1}{2} \tau_{n}\left|\underline{\nabla} \cdot \underline{v}^{h}\right|_{0, \Omega}^{2}+\left(k\left|\underline{v}^{h}\right|_{\varepsilon}, 1\right)^{h}-\left(G_{\varepsilon}^{n}, \underline{\nabla} \cdot \underline{v}^{h}\right)\right\} .
$$

The desired existence and uniqueness results for $Q_{\varepsilon}^{n}$ and $W_{\varepsilon}^{n}$ follow from this and $(2.10)$.

We note that for all $\underline{a} \in \mathbb{R}^{2}$,

$$
|\underline{a}| \leqslant|\underline{a}|_{\varepsilon} \leqslant|\underline{a}|+\varepsilon \quad \text { and } \quad|\underline{a}| \leqslant \frac{|\underline{a}|^{2}+\varepsilon|\underline{a}|}{|\underline{a}|_{\varepsilon}} \leqslant \frac{|\underline{a}|^{2}}{|\underline{a}|_{\varepsilon}}+\varepsilon .
$$

In addition, it follows that for all $\underline{a}, \underline{b} \in \mathbb{R}^{2}$,

$$
\left(\frac{\underline{a}}{|\underline{a}|_{\varepsilon}}-\frac{\underline{b}}{|\underline{b}|_{\varepsilon}}\right) \cdot \underline{a} \geqslant \frac{|\underline{a}|_{\varepsilon}|\underline{b}|_{\varepsilon}-|\underline{a}||\underline{b}|}{|\underline{b}|_{\varepsilon}}-\frac{\varepsilon^{2}}{|\underline{a}|_{\varepsilon}} \geqslant \varepsilon^{2}\left[\frac{1}{|\underline{b}|_{\varepsilon}}-\frac{1}{|\underline{a}|_{\varepsilon}}\right] .
$$

THEOREM 2.1 Let the assumptions (A1) and (A2) hold. Then for all $\varepsilon>0$, for all regular partitionings $\mathcal{T}^{h}$ of $\Omega$, and for all time partitions $\left\{\tau_{n}\right\}_{n=1}^{N}$, the unique solution $\left\{Q_{\varepsilon}^{n}\right\}_{n=1}^{N}$ to $\left(\mathrm{Q}_{\varepsilon}^{h, \tau}\right)$ is such that

$$
\begin{aligned}
& \sum_{n=1}^{N} \tau_{n}\left|\underline{\nabla} \cdot \underline{Q}_{\varepsilon}^{n}\right|_{0, \Omega}^{2} \leqslant 4|k|_{0, \infty, \Omega} \varepsilon|\Omega|+\int_{0}^{T}|f(t)|_{0, \Omega}^{2} \mathrm{~d} t, \\
& \max _{n=1 \rightarrow N}\left|\underline{\nabla} \cdot \underline{U}_{\varepsilon}^{n}\right|_{0, \Omega}^{2}+\sum_{n=1}^{N}\left|\underline{\nabla} \cdot\left(\underline{U}_{\varepsilon}^{n}-\underline{U}_{\varepsilon}^{n-1}\right)\right|_{0, \Omega}^{2}+2 \sum_{n=1}^{N} \tau_{n}\left|k \underline{Q}_{\varepsilon}^{n}\right|_{0,1, \Omega} \\
& \leqslant 4|k|_{0, \infty, \Omega} \varepsilon T|\Omega|+2 \sum_{n=1}^{N} \tau_{n}\left[\left|\bar{f}^{n}\right|_{0, \Omega}^{2}+\left|\underline{\nabla} \cdot \underline{Q}_{\varepsilon}^{n}\right|_{0, \Omega}^{2}\right] .
\end{aligned}
$$

Proof. For $n \geqslant 2$, 2.9a can be rewritten as

$$
\tau_{n}\left(\underline{\nabla} \cdot \underline{Q}_{\varepsilon}^{n}, \underline{\nabla} \cdot \underline{v}^{h}\right)+\left(\frac{k \underline{Q}_{\varepsilon}^{n}}{\left|\underline{Q}_{\varepsilon}^{n}\right|_{\varepsilon}}-\frac{k \underline{Q}_{\varepsilon}^{n-1}}{\left|\underline{Q}_{\varepsilon}^{n-1}\right|_{\varepsilon}}, \underline{v}^{h}\right)^{h}=\left(\int_{t_{n-1}}^{t_{n}} f(t) \mathrm{d} t, \underline{\nabla} \cdot \underline{v}^{h}\right) \quad \forall \underline{v}^{h} \in \underline{V}^{h} .
$$

Choosing $\underline{v}^{h}=Q_{\varepsilon}^{n}$ in 2.15 , and noting 2.13 , implies for $n \geqslant 2$ that

$$
\begin{aligned}
\tau_{n}\left|\underline{\nabla} \cdot \underline{Q}_{\varepsilon}^{n}\right|_{0, \Omega}^{2}+ & \varepsilon^{2}\left(k\left|\underline{Q}_{\varepsilon}^{n-1}\right|_{\varepsilon}^{-1}, 1\right)^{h} \\
& \leqslant \varepsilon^{2}\left(k\left|\underline{Q}_{\varepsilon}^{n}\right|_{\varepsilon}^{-1}, 1\right)^{h}+\frac{1}{2} \tau_{n}\left|\underline{\nabla} \cdot \underline{Q}_{\varepsilon}^{n}\right|_{0, \Omega}^{2}+\frac{1}{2} \tau_{n}^{-1}\left|\int_{t_{n-1}}^{t_{n}} f(t) \mathrm{d} t\right|_{0, \Omega}^{2} \\
& \leqslant \varepsilon^{2}\left(k\left|\underline{Q}_{\varepsilon}^{n}\right|_{\varepsilon}^{-1}, 1\right)^{h}+\frac{1}{2} \tau_{n}\left|\underline{\nabla} \cdot \underline{Q}_{\varepsilon}^{n}\right|_{0, \Omega}^{2}+\frac{1}{2} \int_{t_{n-1}}^{t_{n}}|f(t)|_{0, \Omega}^{2} \mathrm{~d} t .
\end{aligned}
$$


Similarly, choosing $\underline{v}^{h}=\underline{Q}_{\varepsilon}^{1}$ in 2.9 a for $n=1$ yields, on noting 2.8 and 2.12 ,

$$
\begin{aligned}
\tau_{1}\left|\underline{\nabla} \cdot \underline{Q}_{\varepsilon}^{1}\right|_{0, \Omega}^{2} & \leqslant-\left(\frac{k\left|\underline{Q}_{\varepsilon}^{1}\right|^{2}}{\left|\underline{Q}_{\varepsilon}^{1}\right|_{\varepsilon}}, 1\right)^{h}+\left|\underline{\nabla} w^{0}\right|_{0, \infty, \Omega}\left|\underline{Q}_{\varepsilon}^{1}\right|_{0,1, \Omega}+\frac{1}{2} \tau_{1}\left|\underline{\nabla} \cdot \underline{Q}_{\varepsilon}^{1}\right|_{0, \Omega}^{2}+\frac{1}{2} \int_{t_{0}}^{t_{1}}|f(t)|_{0, \Omega}^{2} \mathrm{~d} t \\
& \leqslant 2|k|_{0, \infty, \Omega} \varepsilon|\Omega|+\int_{t_{0}}^{t_{1}}|f(t)|_{0, \Omega}^{2} \mathrm{~d} t
\end{aligned}
$$

Then summing (2.16) for $n=2 \rightarrow N$ and combining with 2.17) yields the desired result 2.14a.

Choosing $\underline{v}^{h}=\underline{Q}_{\varepsilon}^{n}$ in $2.9 \mathrm{a}$, and noting $2.9 \mathrm{~b}$, gives for $n=1 \rightarrow N$ that

$$
\left(\underline{\nabla} \cdot \underline{U}_{\varepsilon}^{n}, \underline{\nabla} \cdot\left(\underline{U}_{\varepsilon}^{n}-\underline{U}_{\varepsilon}^{n-1}\right)\right)+\tau_{n}\left(\frac{k\left|\underline{Q}_{\varepsilon}^{n}\right|^{2}}{\left|\underline{Q}_{\varepsilon}^{n}\right|_{\varepsilon}}, 1\right)^{h}=\tau_{n}\left(\bar{f}^{n}, \underline{\nabla} \cdot \underline{Q}_{\varepsilon}^{n}\right) .
$$

Summing 2.18) from $n=1 \rightarrow \ell$, and recalling the simple identity

$$
2 a(a-b)=\left(a^{2}-b^{2}\right)+(a-b)^{2},
$$

shows for $\ell=1 \rightarrow N$ that

$$
\begin{aligned}
& \frac{1}{2}\left|\underline{\nabla} \cdot \underline{U}_{\varepsilon}^{\ell}\right|_{0, \Omega}^{2}+\frac{1}{2} \sum_{n=1}^{\ell}\left|\underline{\nabla} \cdot\left(\underline{U}_{\varepsilon}^{n}-\underline{U}_{\varepsilon}^{n-1}\right)\right|_{0, \Omega}^{2}+\sum_{n=1}^{\ell} \tau_{n}\left(\frac{k\left|\underline{Q}_{\varepsilon}^{n}\right|^{2}}{\left|\underline{Q}_{\varepsilon}^{n}\right|_{\varepsilon}}, 1\right)^{h} \\
& \quad=\frac{1}{2}\left|\underline{\nabla} \cdot \underline{U}_{\varepsilon}^{0}\right|_{0, \Omega}^{2}+\sum_{n=1}^{\ell} \tau_{n}\left(\bar{f}^{n}, \underline{\nabla} \cdot \underline{Q}_{\varepsilon}^{n}\right) \leqslant \frac{1}{2} \sum_{n=1}^{\ell} \tau_{n}\left[\left|\bar{f}^{n}\right|_{0, \Omega}^{2}+\left|\underline{\nabla} \cdot \underline{Q}_{\varepsilon}^{n}\right|_{0, \Omega}^{2}\right] .
\end{aligned}
$$

Hence the desired result 2.14b follows from 2.20, 2.12) and 2.8.

\section{Convergence of $\mathrm{Q}_{\varepsilon}^{h, \tau}$-existence theory for $(\mathrm{Q})$}

Let

$$
\underline{Q}_{\varepsilon}^{+}(t):=\underline{Q}_{\varepsilon}^{n}, \quad \underline{U}_{\varepsilon}^{+}(t):=\underline{U}_{\varepsilon}^{n}, \quad W_{\varepsilon}^{+}(t):=W_{\varepsilon}^{n}, \quad \bar{f}^{+}(t):=\bar{f}^{n} \quad t \in\left(t_{n-1}, t_{n}\right], \quad n \geqslant 1 .
$$

It follows from (3.1) and 2.9b that for $t \in\left(t_{n-1}, t_{n}\right], n \geqslant 1$,

$$
\begin{aligned}
\left|\underline{\nabla} \cdot\left(\underline{U}_{\varepsilon}^{+}(t)-\int_{0}^{t} \underline{Q}_{\varepsilon}^{+}(r) \mathrm{d} r\right)\right|_{0, \Omega} & =\left|\int_{t}^{t_{n}} \underline{\nabla} \cdot \underline{Q}_{\varepsilon}^{+}(r) \mathrm{d} r\right|_{0, \Omega} \\
& \leqslant\left(\tau_{n}\right)^{1 / 2}\left(\int_{t_{n-1}}^{t_{n}}\left|\underline{\nabla} \cdot \underline{Q}_{\varepsilon}^{+}\right|_{0, \Omega}^{2} \mathrm{~d} t\right)^{1 / 2} .
\end{aligned}
$$

Similarly, we have

$$
\left|\bar{f}^{+}(t)-\bar{f}(t)\right|_{0, \Omega} \leqslant\left(\tau_{n}\right)^{1 / 2}\left(\int_{t_{n-1}}^{t_{n}}|f|_{0, \Omega}^{2} \mathrm{~d} t\right)^{1 / 2} \quad \text { for } t \in\left(t_{n-1}, t_{n}\right], n \geqslant 1 .
$$


Adopting the notation $\sqrt{3.1},\left(\mathrm{Q}_{\varepsilon}^{h, \tau}\right)$ can be restated as:

$$
\begin{aligned}
\int_{0}^{T}\left[\left(\underline{\nabla} \cdot \underline{U}_{\varepsilon}^{+}-\bar{f}^{+}, \underline{\nabla} \cdot \underline{v}^{h}\right)+\left(\frac{k \underline{Q}_{\varepsilon}^{+}}{\left|\underline{Q}_{\varepsilon}^{+}\right|_{\varepsilon}}, \underline{v}^{h}\right)^{h}\right] \mathrm{d} t=0 & \forall \underline{v}^{h} \in L^{\infty}\left(0, T ; \underline{V}^{h}\right) ; \\
\int_{0}^{T}\left(W_{\varepsilon}^{+}-\bar{f}^{+}+\underline{\nabla} \cdot \underline{U}_{\varepsilon}^{+}, \eta^{h}\right) \mathrm{d} t=0 & \forall \eta^{h} \in L^{\infty}\left(0, T ; S^{h}\right) .
\end{aligned}
$$

THEOREM 3.1 Let the assumptions (A1) and (A2) hold. For all regular partitionings $\mathcal{T}^{h}$ of $\Omega$, and for all time partitions $\left\{\tau_{n}\right\}_{n=1}^{N}$ and for all $\varepsilon>0$ such that $\tau, \varepsilon \rightarrow 0$ as $h \rightarrow 0$, the unique solution $\left\{\underline{Q}_{\varepsilon}^{n}\right\}_{n=1}^{N}$ to $\left(\mathrm{Q}_{\varepsilon}^{h, \tau}\right)$ is such that there exists a subsequence of $\left\{\underline{Q}_{\varepsilon}^{+}\right\}_{h}$, where $\underline{Q}_{\varepsilon}^{+}$solves $\left(\mathrm{Q}_{\varepsilon}^{h, \tau}\right)$, and a $\underline{q} \in \underline{\mathcal{M}}$ (div) such that as $h \rightarrow 0$,

$$
\begin{aligned}
& \left.\underline{Q}_{\varepsilon}^{+}\right|_{\Omega_{T}^{(i)}} \rightarrow \underline{q}^{(i)} \quad \text { weakly in }\left[\mathcal{M}\left(\Omega_{T}^{(i)}\right)\right]^{2}, i=1 \rightarrow I, \\
& \underline{\nabla} \cdot \underline{Q}_{\varepsilon}^{+} \rightarrow \underline{\nabla} \cdot \underline{q} \quad \text { weakly in } L^{2}\left(\Omega_{T}\right) \text {, } \\
& \underline{\nabla} \cdot \underline{U}_{\varepsilon}^{+} \rightarrow \underline{\nabla} \cdot \underline{u} \quad \text { weak- } \star \text { in } L^{\infty}\left(0, T ; L^{2}(\Omega)\right), \\
& W_{\varepsilon}^{+} \rightarrow w \quad \text { weak- } \star \text { in } L^{\infty}\left(0, T ; L^{2}(\Omega)\right) \text {, }
\end{aligned}
$$

where

$$
\underline{\nabla} \cdot \underline{u}(t):=\underline{\nabla} \cdot\left(\int_{0}^{t} \underline{q}(r) \mathrm{d} r\right) \text { and } \quad w(t):=\int_{0}^{t} f(r) \mathrm{d} r+w^{0}-\underline{\nabla} \cdot \underline{u}(t) .
$$

Moreover, $\underline{q}$ solves $(\mathrm{Q}), 2.1$.

Proof. The bounds 2.14a b) yield, on noting (3.1) and (1.4,

$\left\|\underline{\nabla} \cdot \underline{Q}_{\varepsilon}^{+}\right\|_{L^{2}\left(\Omega_{T}\right)}^{2}+\left\|\underline{Q}_{\varepsilon}^{+}\right\|_{L^{1}\left(\Omega_{T}\right)}+\left\|\underline{\nabla} \cdot \underline{U}_{\varepsilon}^{+}\right\|_{L^{\infty}\left((0, T), L^{2}(\Omega)\right)} \leqslant C(T, k,|\Omega|)\left[1+\|f\|_{L^{2}\left(\Omega_{T}\right)}^{2}\right]$.

The subsequence convergence results 3.5a-d) then follow immediately from 3.7, , 1.19), 3.2, (3.4b) and 3.3.

Let $\underline{I}^{h}:\left[H^{1}(\Omega)\right]^{2} \rightarrow \underline{V}^{h}$ be the generalised interpolation operator satisfying

$$
\int_{\partial_{i} \sigma}\left(\underline{v}-\underline{I}^{h} \underline{v}\right) \cdot \underline{n}_{\partial_{i} \sigma}=0, \quad i=1 \rightarrow 3, \forall \sigma \in \mathcal{T}^{h}
$$

where $\partial \sigma \equiv \bigcup_{i=1}^{3} \partial_{i} \sigma$ is the boundary of $\sigma$ and $\underline{n}_{\partial_{i}} \sigma$ are the corresponding outward unit normals on $\partial_{i} \sigma$. It follows (see e.g. [16, 553]) that for all $\sigma \in \mathcal{T}^{h}$,

$$
\left|\underline{v}-\underline{I}^{h} \underline{v}\right|_{0, \sigma} \leqslant C h|\underline{v}|_{1, \sigma},
$$

and if $\underline{v}$ is sufficiently smooth,

$$
\left|\underline{\nabla} \cdot\left(\underline{v}-\underline{I}^{h} \underline{v}\right)\right|_{0, \sigma} \leqslant C h|\underline{\nabla} \cdot \underline{v}|_{1, \sigma} \quad \text { and } \quad\left|\underline{I}^{h} \underline{v}\right|_{1, \sigma} \leqslant C\|\underline{v}\|_{2, \sigma} .
$$


In addition, we note from 2.3 and 2.7 that for all $\sigma \in \mathcal{T}^{h}$,

$$
\begin{aligned}
\left.\left|\int_{\sigma}\right| \underline{v}\right|_{\varepsilon}-\left(\left|\underline{I}^{h} \underline{v}\right|_{\varepsilon}, 1\right)_{\sigma}^{h} \mid & \leqslant\left.\int_{\sigma}|| \underline{v}\right|_{\varepsilon}-\left|\underline{I}^{h} \underline{v}\right|_{\varepsilon}|+| \int_{\sigma}\left|\underline{I}^{h} \underline{v}\right|_{\varepsilon}-\left(\left|\underline{I}^{h} \underline{v}\right|_{\varepsilon}, 1\right)_{\sigma}^{h} \mid \\
& \leqslant\left|\underline{v}-\underline{I}^{h} \underline{v}\right|_{0,1, \sigma}+\left.\left.C h|\sigma||| \underline{I}^{h} \underline{v}\right|_{\varepsilon}\right|_{1, \infty, \sigma} \\
& \leqslant\left|\underline{v}-\underline{I}^{h} \underline{v}\right|_{0,1, \sigma}+C h\left|I^{h} \underline{v}\right|_{1,1, \sigma} .
\end{aligned}
$$

Given any $\underline{v} \in\left[C^{\infty}\left(\bar{\Omega}_{T}\right)\right]^{2}$, we choose $\underline{v}^{h} \equiv \underline{Q}_{\varepsilon}^{+}-\underline{I}^{h} \underline{v}$ in $3.4 \mathrm{a}$. Hence, on noting 2.4 , we have

$$
\begin{aligned}
\int_{0}^{T}\left[\left(\underline{\nabla} \cdot \underline{U}_{\varepsilon}^{+}-\bar{f}^{+}, \underline{\nabla} \cdot\left[\underline{I}^{h} \underline{v}\right]\right)+\right. & \left.\left(k\left|\underline{I}^{h} \underline{v}\right|_{\varepsilon}, 1\right)^{h}\right] \mathrm{d} t \\
& \geqslant \int_{0}^{T}\left[\left(\underline{\nabla} \cdot \underline{U}_{\varepsilon}^{+}-\bar{f}^{+}, \underline{\nabla} \cdot \underline{Q}_{\varepsilon}^{+}\right)+\left(k\left|\underline{Q}_{\varepsilon}^{+}\right|_{\varepsilon}, 1\right)^{h}\right] \mathrm{d} t .
\end{aligned}
$$

It follows immediately from $3.5 \mathrm{~b}, \mathrm{c}), 3.9 \mathrm{~b}$ and $3.3 \mathrm{~b}$ that

$$
\begin{aligned}
\int_{0}^{T}\left[\left(\underline{\nabla} \cdot \underline{U}_{\varepsilon}^{+}-\bar{f}^{+}, \underline{\nabla} \cdot\left[\underline{I}^{h} \underline{v}\right]\right)\right. & \left.+\left(\bar{f}^{+}, \underline{\nabla} \cdot \underline{Q}_{\varepsilon}^{+}\right)\right] \mathrm{d} t \\
\rightarrow & \int_{0}^{T}[(\underline{\nabla} \cdot \underline{u}-\bar{f}, \underline{\nabla} \cdot \underline{v})+(\bar{f}, \underline{\nabla} \cdot \underline{q})] \mathrm{d} t \quad \text { as } h \rightarrow 0 .
\end{aligned}
$$

Next we note from 2.5, 2.12, 2.8, 23.5a) and 1.20) that for $i=1 \rightarrow I$,

$$
\begin{aligned}
\liminf _{h \rightarrow 0} \int_{0}^{T} \sum_{\sigma \in \mathcal{T}_{i}^{h}}\left(\left|\underline{Q}_{\varepsilon}^{+}\right|_{\varepsilon}, 1\right)_{\sigma}^{h} \mathrm{~d} t & \geqslant \liminf _{h \rightarrow 0} \int_{0}^{T} \sum_{\sigma \in \mathcal{T}_{i}^{h}}\left(\left|\underline{Q}_{\varepsilon}^{+}\right|, 1\right)_{\sigma}^{h} \mathrm{~d} t \\
& \geqslant \liminf _{h \rightarrow 0} \int_{\Omega_{T}^{(i)}}\left|\underline{Q}_{\varepsilon}^{+}\right| \geqslant \int_{\Omega_{T}^{(i)}}\left|\underline{q}^{(i)}\right| .
\end{aligned}
$$

It follows from 2.5, 2.12, 3.10 and 3.9a, b) that for $i=1 \rightarrow I$,

$$
\lim _{h \rightarrow 0} \int_{0}^{T} \sum_{\sigma \in \mathcal{T}_{i}^{h}}\left(\left|\underline{I}^{h} \underline{v}\right|_{\varepsilon}, 1\right)_{\sigma}^{h} \mathrm{~d} t=\int_{\Omega_{T}^{(i)}}|\underline{v}| .
$$

Let

$$
\underline{U}_{\varepsilon}(t):=\frac{t_{n}-t}{\tau_{n}} \underline{U}_{\varepsilon}^{n-1}+\frac{t-t_{n-1}}{\tau_{n}} \underline{U}_{\varepsilon}^{n}, \quad t \in\left[t_{n-1}, t_{n}\right], n=1 \rightarrow N .
$$

Similarly to 3.2), it follows from 3.1], 2.9b) and 3.7p that

$$
\int_{0}^{T}\left|\underline{\nabla} \cdot\left(\underline{U}_{\varepsilon}^{+}-\underline{U}_{\varepsilon}\right)\right|_{0, \Omega}^{2} \leqslant C \tau^{2} \quad \text { and } \quad \partial_{t} \underline{U}_{\varepsilon}=\underline{Q}_{\varepsilon}^{+} \quad \text { a.e. in } \Omega_{T} .
$$


Hence from (3.16), 3.7), 2.9b, 3.5c) and (3.6) we have

$$
\begin{aligned}
\liminf _{h \rightarrow 0} \int_{0}^{T}\left(\underline{\nabla} \cdot \underline{U}_{\varepsilon}^{+}, \underline{\nabla} \cdot \underline{Q}_{\varepsilon}^{+}\right) \mathrm{d} t & =\liminf _{h \rightarrow 0} \int_{0}^{T}\left(\underline{\nabla} \cdot \underline{U_{\varepsilon}}, \underline{\nabla} \cdot \underline{Q}_{\varepsilon}^{+}\right) \mathrm{d} t=\frac{1}{2} \liminf _{h \rightarrow 0}\left|\underline{\nabla} \cdot \underline{U_{\varepsilon}}(T)\right|_{0, \Omega}^{2} \\
& \geqslant \frac{1}{2}|\underline{\nabla} \cdot \underline{u}(T)|_{0, \Omega}^{2}=\int_{0}^{T}(\underline{\nabla} \cdot \underline{u}, \underline{\nabla} \cdot \underline{q}) \mathrm{d} t .
\end{aligned}
$$

Combining 3.11 - 3.14 and 3.17, we see that $\underline{q}$ solves 2.1 holds for any $\underline{v} \in\left[C^{\infty}\left(\bar{\Omega}_{T}\right)\right]^{2}$. The desired result, that $\underline{q}$ solves $\{2.1\}$ for any $\underline{v} \in \overline{\widehat{V}}_{\mathcal{M}}(\mathrm{div})$, then follows from the density results $1.21 \mathrm{a}$ c) which clearly hold for $\widehat{\underline{V}}_{\mathcal{M}}$ (div).

THEOREM 3.2 Let the assumptions of Theorem 3.1 hold. Then $w$ defined by 3.6 is such that $w \in L^{\infty}\left(0, T ; K_{0}(k)\right) \cap H^{1}\left(0, T ; L^{2}(\Omega)\right)$ with $w(0)=w^{0}$. Moreover, $w$ solves $(\mathrm{P})$, 1.2 with $K_{w^{0}}(k)$ replaced by $K_{0}(k)$, and is therefore unique. In addition, $w$ and the solution $\underline{q} \in \underline{V}_{\mathcal{M}}$ (div) of (2.1) satisfy (1.13a,b).

Finally, the possible non-uniqueness in $q$ is restricted to the following: If there were two solutions $\underline{q}_{\ell}, \ell=1,2$, then

$$
\underline{\nabla} \cdot\left(\underline{q}_{1}-\underline{q}_{2}\right)=0 \quad \text { a.e. in } \Omega_{T} \quad \text { and } \quad\left\langle k,\left|\underline{q}_{1}\right|\right\rangle=\left\langle k,\left|\underline{q}_{2}\right|\right\rangle .
$$

Proof. It follows from 3.6 and $3.5 \mathrm{~b}$ that $w \in H^{1}\left(0, T ; L^{2}(\Omega)\right)$ satisfies $w(0)=w^{0}$ and $1.13 \mathrm{a}$. Combining 2.1 and 3.6 yields

$$
\langle k,|\underline{v}|\rangle-\langle k,|\underline{q}|\rangle-\int_{0}^{T}(w, \underline{\nabla} \cdot(\underline{v}-\underline{q})) \mathrm{d} t \geqslant 0 \quad \forall \underline{v} \in \underline{\widehat{V}}_{\mathcal{M}}(\operatorname{div}) .
$$

Let $J(v):=\langle k,|\underline{v}|\rangle-\int_{0}^{T}(w, \underline{\nabla} \cdot \underline{v}) \mathrm{d} t$. From 3.19 we obtain

$$
J(\underline{q}) \leqslant \mathcal{J}:=\inf _{\underline{v} \in \underline{\widehat{V}}_{\mathcal{M}}(\mathrm{div})} J(\underline{v}) \leqslant J(0)=0 .
$$

If $\mathcal{J}<0$ then, for any minimizing sequence $\left\{\underline{v}_{j}\right\}$, we obtain $J\left(2 \underline{v}_{j}\right) \rightarrow 2 \mathcal{J}<\mathcal{J}$, which is a contradiction. Hence $\mathcal{J}=0$, and so $\langle k,|\underline{v}|\rangle \geqslant \int_{0}^{T}(w, \underline{\nabla} \cdot \underline{v}) \mathrm{d} t$ for any $\underline{v} \in \underline{\underline{V}}_{\mathcal{M}}(\operatorname{div})$. Since this is true also for $-\underline{v}$, we obtain

$$
\left|\int_{0}^{T}(w, \underline{\nabla} \cdot \underline{v}) \mathrm{d} t\right| \leqslant\langle k,|\underline{v}|\rangle \quad \forall \underline{v} \in \widehat{\underline{V}}_{\mathcal{M}}(\operatorname{div}) .
$$

It immediately follows from 3.21 that $w \in L^{\infty}\left(0, T ; K_{0}(k)\right)$.

Given any $\eta \in L^{\infty}\left(0, T ; K_{0}(k)\right)$, we introduce, for any $\delta \in\left(0, \delta_{0}\right)$,

$$
\eta_{\delta}(\underline{x}, t):=\psi_{\delta}(t)\left([\eta(\underline{x}, t)-\delta]_{+}-[\eta(\underline{x}, t)+\delta]_{-}\right) \quad \text { for a.e. }(\underline{x}, t) \in \Omega_{T},
$$

where $\psi_{\delta} \in C_{0}^{\infty}(0, T), 0 \leqslant \psi_{\delta} \leqslant 1$ and $\psi_{\delta}(t)=1$ if $t \in(\delta, T-\delta)$. It follows that $\eta_{\delta} \in$ $L^{\infty}\left(0, T ; K_{0}(k)\right)$ with compact support in $\Omega_{T}$ such that

$$
\left|\underline{\nabla} \eta_{\delta}(\underline{x}, t)\right| \leqslant|\underline{\nabla} \eta(\underline{x}, t)| \quad \text { for a.e. }(\underline{x}, t) \in \Omega_{T} \quad \text { and } \quad \lim _{\delta \rightarrow 0} \int_{0}^{T}\left\|\eta-\eta_{\delta}\right\|_{1, \Omega} \mathrm{d} t=0 .
$$


In addition, on extending $q^{(i)}, i=1 \rightarrow I$, by zero and applying mollifiers, one can construct $\left\{\underline{q}_{j}\right\}_{j} \geqslant 0$, where $\underline{q}_{j} \equiv \sum_{i=1}^{I} \underline{q}_{j}^{(i)}$ with $\underline{q}_{j}^{(i)} \in\left[C^{\infty}\left(\bar{\Omega}_{T}\right)\right]^{2}, i=1 \rightarrow I$, such that

$$
\begin{aligned}
& \int_{\Omega_{T}}\left|\underline{q}_{j}^{(i)}\right| \leqslant \int_{\Omega_{T}^{(i)}}\left|\underline{q}^{(i)}\right|, \quad i=1 \rightarrow I, \\
& \underline{\nabla} \cdot \underline{q}_{j} \rightarrow \underline{\nabla} \cdot \underline{q} \quad \text { weakly in } L^{2}(\omega) \text { for any } \bar{\omega} \subset \Omega_{T} .
\end{aligned}
$$

Combining 3.23 and $3.24 \mathrm{a} b)$, for any $\eta \in L^{\infty}\left(0, T ; K_{0}(k)\right)$ we have

$$
\begin{aligned}
\int_{0}^{T}(\underline{\nabla} \cdot \underline{q}, \eta) \mathrm{d} t & =\int_{0}^{T}\left[-\left(\underline{q}_{j}, \underline{\nabla} \eta_{\delta}\right)+\left(\underline{\nabla} \cdot\left(\underline{q}-\underline{q}_{j}\right), \eta_{\delta}\right)+\left(\underline{\nabla} \cdot \underline{q}, \eta-\eta_{\delta}\right)\right] \mathrm{d} t \\
& \leqslant \sum_{i=1}^{I} k^{(i)} \int_{\Omega_{T}^{(i)}}\left|\underline{q}^{(i)}\right|+\left|\int_{0}^{T}\left(\underline{\nabla} \cdot\left(\underline{q}-\underline{q}_{j}\right), \eta_{\delta}\right) \mathrm{d} t\right|+C \int_{0}^{T}\left\|\eta-\eta_{\delta}\right\|_{1, \Omega} \mathrm{d} t \\
& \rightarrow\langle k,|\underline{q}|\rangle \quad \text { as } j \rightarrow \infty, \delta \rightarrow 0 .
\end{aligned}
$$

Applying (3.25) for $\eta \equiv w$ and noting that $J(q) \leqslant 0$ yield the desired result $1.13 \mathrm{~b}$. Hence $w$ and the solution $\underline{q} \in \underline{V}_{\mathcal{M}}$ (div) of (2.1) satisfy (1.13ab).

Choosing $\eta \equiv \xi-w$ in 1 .13a with $\xi \in L^{\sim}\left(0, T ; K_{0}(k)\right)$ and noting 3.25 and $1.13 \mathrm{~b}$ ) shows that $w$ solves the primal variational inequality $(\mathrm{P}),(1.2)$ with $K_{w^{0}}(k)$ replaced by $K_{0}(k)$, and is therefore unique.

Finally, combining the uniqueness of $w$ and (3.6) implies that if there were two solutions $q_{\ell} \in$ $\underline{V}_{\mathcal{M}}(\mathrm{div})$ solving $(\mathrm{Q})$, then the first result in 3.18 holds. The second result in $(3.18)$ then follows from the first, the uniqueness of $w$ and $(1.13 \mathrm{~b})$.

We end this section by noting a consequence of $\left(\mathrm{E}_{2}\right)$, and its discrete analogue. Firstly, $\left(\mathrm{E}_{2}\right)$ implies, on choosing $\eta=w$ in $(1.13 \mathrm{a})$ and combining with $(1.13 \mathrm{~b})$, that the following equality holds over the time interval $[0, T]$ :

$$
\frac{1}{2}|w(\cdot, T)|_{0}^{2}+\langle k,|\underline{q}|\rangle=\frac{1}{2}\left|w^{0}\right|_{0}^{2}+\int_{0}^{T}(f, w) \mathrm{d} t .
$$

Of course, one can deduce that a similar equality holds over any time interval $\left[T_{1}, T_{2}\right] \subset[0, T]$. We now derive a discrete analogue of this result. One deduces from 2.10 and $2.9 \mathrm{~b}$ ) that for $n=$ $1 \rightarrow N$,

$$
\left(\frac{W_{\varepsilon}^{n}-W_{\varepsilon}^{n-1}}{\tau_{n}}, \eta^{h}\right)=\left(\frac{1}{\tau_{n}} \int_{t_{n-1}}^{t_{n}} f(r) \mathrm{d} r-\underline{\nabla} \cdot \underline{Q}_{\varepsilon}^{n}, \eta^{h}\right) \quad \forall \eta^{h} \in S^{h},
$$

where we have naturally defined $W_{\varepsilon}^{0} \in S^{h}$ by the projection $\left(W_{\varepsilon}^{0}-w^{0}, \eta^{h}\right)=0$ for all $\eta^{h} \in S^{h}$. On choosing $\underline{v}^{h}=\underline{Q}_{\varepsilon}^{n}$ in $2.9 \mathrm{a}$, and noting $2.9 \mathrm{~b}$ and 2.10 , we see that for $n=1 \rightarrow N$,

$$
\left(k \frac{\left|\underline{Q}_{\varepsilon}^{n}\right|^{2}}{\left|\underline{Q}_{\varepsilon}^{n}\right|_{\varepsilon}}, 1\right)^{h}=\left(\bar{f}^{n}-\underline{\nabla} \cdot \underline{U}_{\varepsilon}^{n}, \underline{\nabla} \cdot \underline{Q}_{\varepsilon}^{n}\right)=\left(W_{\varepsilon}^{n}, \underline{\nabla} \cdot \underline{Q}_{\varepsilon}^{n}\right) .
$$


Therefore 3.27a b) are the discrete analogues of $1.13 \mathrm{a}$ b). Finally, choosing $\eta^{h}=W_{\varepsilon}^{n}$ in $3.27 \mathrm{a}$ and combining with $3.27 \mathrm{~b}$ implies for $n=1 \rightarrow N$ that

$$
\frac{1}{2}\left[\left|W_{\varepsilon}^{n}\right|_{0}^{2}+\left|W_{\varepsilon}^{n}-W_{\varepsilon}^{n-1}\right|_{0}^{2}\right]+\tau_{n}\left(k \frac{\left|\underline{Q}_{\varepsilon}^{n}\right|^{2}}{\left|\underline{Q}_{\varepsilon}^{n}\right|_{\varepsilon}}, 1\right)^{h}=\frac{1}{2}\left|W_{\varepsilon}^{n-1}\right|_{0}^{2}+\left(\int_{t_{n-1}}^{t_{n}} f(r) \mathrm{d} r, W_{\varepsilon}^{n}\right)
$$

which is the discrete analogue of 3.26 .

\section{Numerical experiments}

At each time level $n$, the resulting non-linear algebraic system 2.9a was solved iteratively using the following successive over-relaxation algorithm:

$$
\begin{aligned}
\text { Given } \underline{Q}_{\varepsilon}^{n, 0} \in \underline{V}^{h} \text {, for } j \geqslant 0 \text { find } \underline{Q}_{\varepsilon}^{n, j+1 / 2} \in \underline{V}^{h} \text { such that } \\
\tau_{n}\left(\underline{\nabla} \cdot \underline{Q}_{\varepsilon}^{n, j+1 / 2}, \underline{\nabla} \cdot \underline{v}^{h}\right)+\left(\frac{k \underline{Q}_{\varepsilon}^{n, j+1 / 2}}{\left|\underline{Q}_{\varepsilon}^{n, j}\right|_{\varepsilon}}, \underline{v}^{h}\right)^{h}=\left(G_{\varepsilon}^{n}, \underline{\nabla} \cdot \underline{v}^{h}\right) \quad \forall \underline{v}^{h} \in \underline{V}^{h},
\end{aligned}
$$

and set

$$
\underline{Q}_{\varepsilon}^{n, j+1}=\gamma \underline{Q}_{\varepsilon}^{n, j+1 / 2}+(1-\gamma) \underline{Q}_{\varepsilon}^{n, j} .
$$

Clearly the above is a well-posed algorithm, involving the solution of a symmetric positive definite linear system at each iteration. When the iterations converged up to a prescribed tolerance, we set $\underline{Q}_{\varepsilon}^{n}=\underline{Q}_{\varepsilon}^{n, j+1}$ and then recovered the primary variable $W_{\varepsilon}^{n}$ explicitly from 2.10 . For $n \geqslant 1$, we chose $\underline{Q}_{\varepsilon}^{n, 0}=\underline{Q}_{\varepsilon}^{n-1}$ and observed good convergence properties of this algorithm. In all of our simulations we set $\varepsilon=10^{-7}$; the relaxation parameter $\gamma=1.3$; and performed the iteration $4.1 \mathrm{a}$ b) until either $\left|Q_{\varepsilon}^{n, j+1}-\underline{Q}_{\varepsilon}^{n, j}\right|_{0, \infty} \leqslant$ tol or $\left|\underline{Q}_{\varepsilon}^{n, j+1}-\underline{Q}_{\varepsilon}^{n, j}\right|_{0, \infty} /\left|\underline{Q}_{\varepsilon}^{n, j+1}\right|_{0, \infty} \leqslant t o l$, where $t o l$ is either $10^{-4}$ or $10^{-5}$. Typically, approximately fifty iterations per time step were sufficient. However, if the solution changed drastically during a particular time step, then more iterations could be required to meet the prescribed tolerance. Neither the solution nor the number of iterations were sensitive to the value of $\varepsilon$. In all of the examples below we assumed a zero initial state and used a fixed time step $\tau \in[0.0125,0.05]$. The Matlab PDE Toolbox [11] was employed for the domain triangulation; domains with curved boundaries were approximated by polygons. We refer to [3] for the Matlab implementation of the Raviart-Thomas element of the lowest order.

In contrast to algorithms based on primal variational formulations of critical-state problems, the dual formulation allows one to approximate both the primal and dual variables simultaneously. Moreover, our numerical simulations (see below) seem to indicate that, although different algorithms can be employed to solve the arising variational problems, even the primal variables are possibly easier to compute using the dual formulation. For these primal variables comparison with the analytical solution, if known, showed first order convergence in both $h$ and $\tau$ in the $L^{\infty}\left(\Omega_{T}\right)$ norm. The first two examples illustrate known analytical solutions of the sand problem (see Section 11); here we set $k \equiv 1$.

Numerical simulation of a growing conical pile is presented in Fig. 1, where $\Omega$ is a unit square; a regular $60 \times 60$ grid was used for its triangulation. The surface flux $q$ in this example has a $|\underline{x}|^{-1}$ singularity before the discharge time, when the conical pile meets the boundary, and 

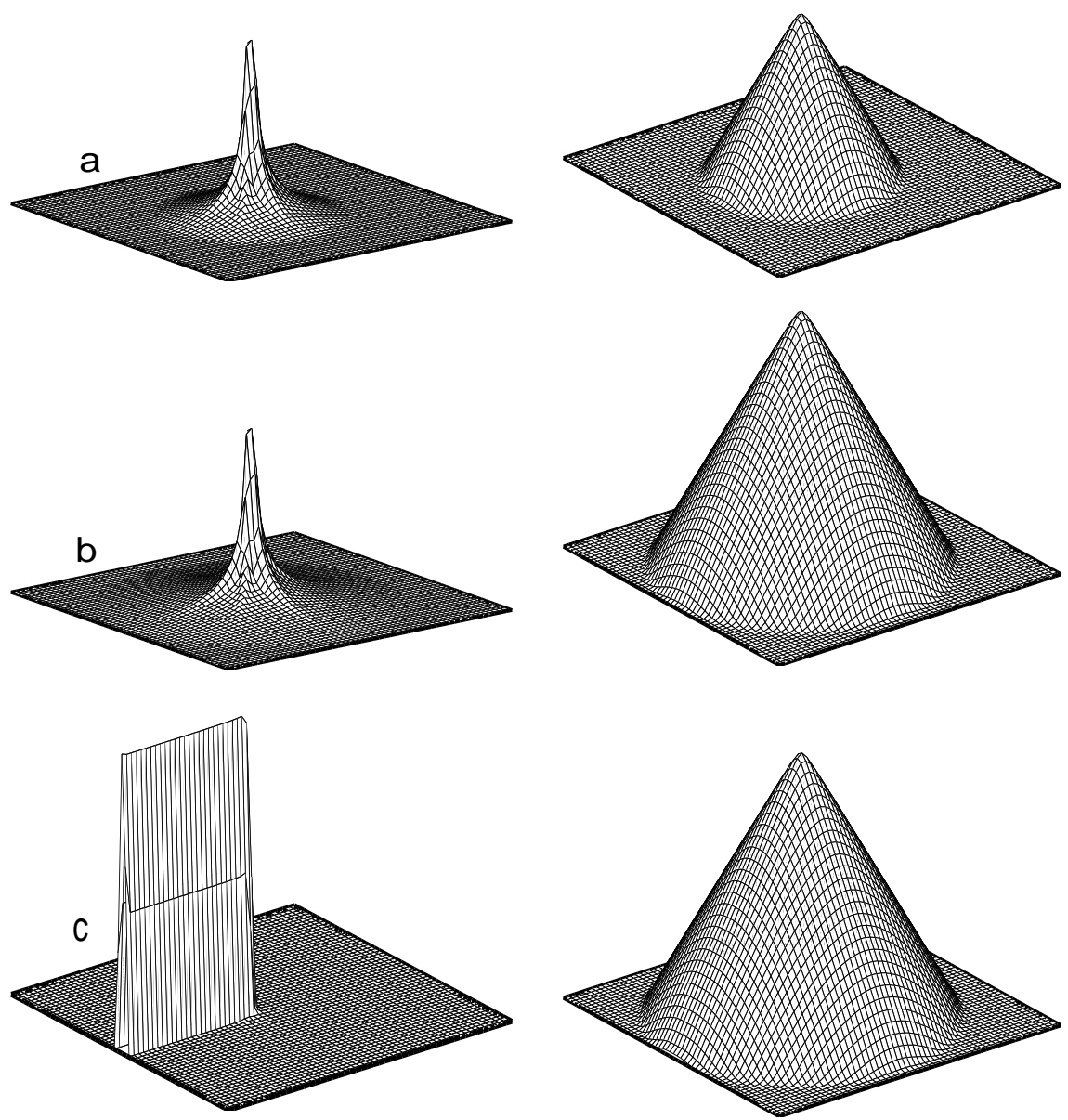

FIG. 1. Pile on a square open support growing under a point source $f=0.2 \delta\left(\underline{x}-\underline{x}_{0}\right)$. The cone grows (a) until its base touches the support boundary, (b) just before this moment. Then a runway appears and the pile growth stops, (c) after this moment. Shown: left—sand flux $\left|Q_{\varepsilon}\right|$, right—the pile surface computed using 2.10 .

is a measure concentrated along the runway when the latter appears. We note that our stability and convergence analysis in the previous sections assumes that $f \in L^{2}\left(\Omega_{T}\right)$, and thus does not cover the case of a point source. Nevertheless, the numerical approximation $\left(\mathrm{Q}_{\varepsilon}^{h, \tau}\right)$ is still well-defined, when the point source is placed in the interior of an element and we placed the source at the center of an element. The resulting numerical solution is qualitatively very good: as can be seen in Fig. 11. $Q_{\varepsilon}$ captures the singular behavior in $q$ remarkably well, and is inversely proportional to the mesh size in the neighborhood of these singularities. The accuracy of the primal variable, $W_{\varepsilon}$, is easy to calculate; for $\tau=0.025$ and the stated mesh we found that $\left\{\max _{n}\left|w\left(\cdot, t_{n}\right)-W_{\varepsilon}\left(\cdot, t_{n}\right)\right|_{0, \infty, \Omega}\right\} /|w|_{0, \infty, \Omega_{T}} \leqslant 0.014$. Since the surface $W_{\varepsilon}$ is found from the surface flux $Q_{\varepsilon}$, using 2.10 , this indirectly characterizes also the error in $Q_{\varepsilon}$.

As stated above, after the discharge time the conical pile, $w$, remains steady and the flux $q$ is singular along the straight runway. In addition, one has $\int_{\Omega}|q|=(f, w)$, which can also be 

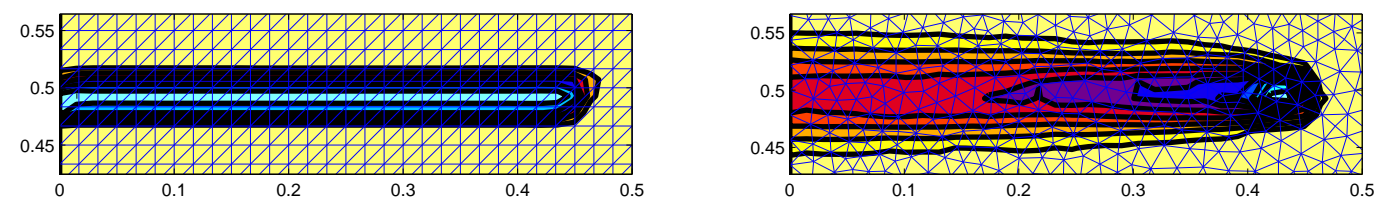

FIG. 2. Contour plots of $\left|Q_{\varepsilon}\right|$ in a neighborhood of the runway, numerical simulation on two different grids. Smearing of the runway is weaker for the regular mesh (left) than for the general mesh not adapted to the runway (right).

deduced from 3.26 . We note that the use of a regular grid helps in the approximation of $q$ at this stage, as the finite elements are aligned with the runway. Computing on a non-regular mesh with the same number of elements resulted in the runway being smeared across several elements (see Fig. 2); the error in $W_{\varepsilon}$ was also doubled on this non-regular mesh. We note from (3.28) that although the runway may be smeared, the strength of the singularity is still well approximated. This mesh-determined smearing effect is clearly seen in two other numerical examples below (see Figs. 5 and 9p in which the dual variable is a vectorial measure partly supported on a subset of dimension one, to which the mesh is not specially adapted. Nevertheless, qualitatively the singular behavior of this variable is satisfactory captured also in these examples. Of course, adaptive mesh refinement would increase the accuracy of the computed solution, and this will be an area of future study.

The second example illustrates the distributed source case (see Fig. 3 for the geometric configuration). The growing pile is a truncated cone until its base first touches the domain boundary; the growth ends when all points in the support of $f$ become connected to the border by a straight transport ray through which the discharged sand leaves the system (Fig. (4).

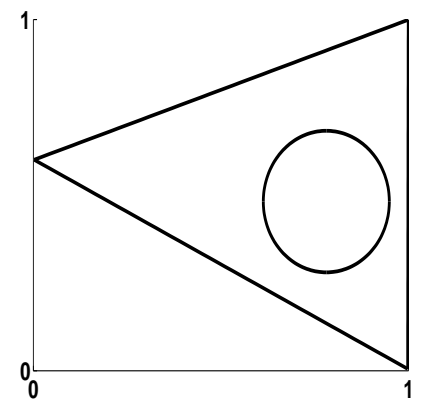

FIG. 3. Triangular support was divided into 4080 finite elements (maximal element diameter $h=0.0167$ ). The source: $f=1$ inside the ellipse and $f=0$ outside.

Suppose now the support domain is partly surrounded by a vertical impermeable wall. Then on the closed part of the support boundary, the open boundary condition in (1.1) should be replaced by the condition $q_{n}=0$. The wall presents an obstacle to the sand flow and the flux $q$ can become a measure concentrated partly along the obstacle boundary [8]; this can happen even if the source is distributed. Similarly to [15], we can transform this situation into our framework by extending the domain beyond the wall and setting $k=\infty$, or to be very large, in this extension. In our next example (see Fig. 5. left), $k=1$ inside a multiply connected domain (white area). The grey area is the extension of this domain beyond the closed part of its boundary and there we set $k=10^{6}$. The 

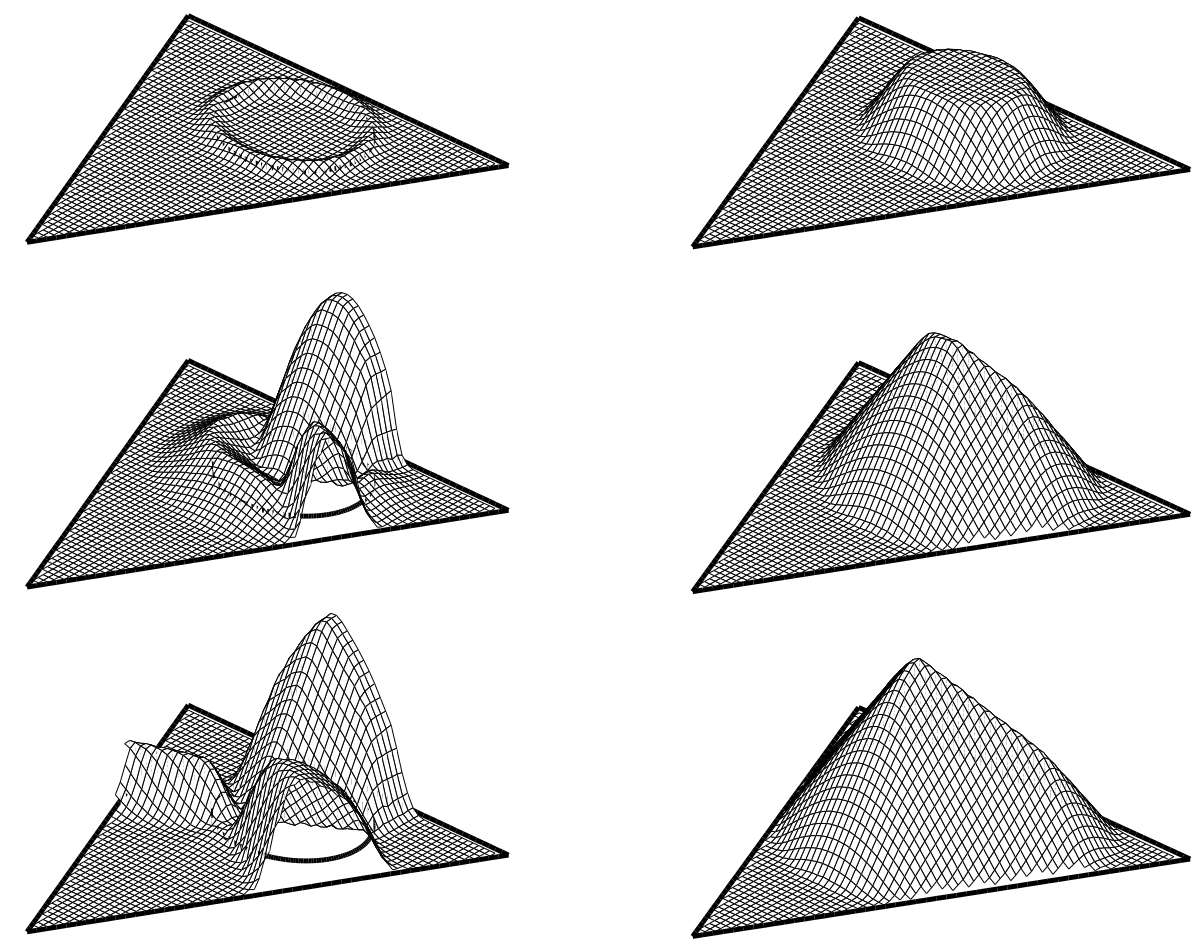

FIG. 4. Pile growing on a triangular support under a distributed source (see Fig. 33. The pile shape stabilises when all points in $\operatorname{supp}(f)$ become connected to the border by a transport ray. Shown: left—sand flux $\left|Q_{\varepsilon}\right|$, right—-the pile surface $W_{\varepsilon}$.
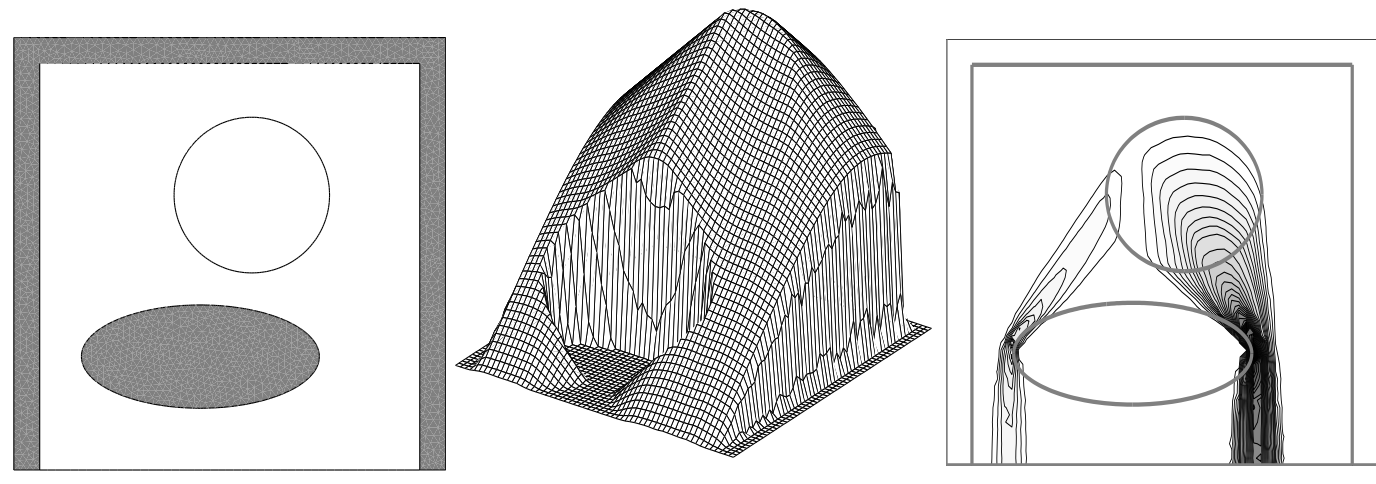

FIG. 5. Pile on a support partly surrounded by a wall. Left-the domain (white region) is extended beyond the impermeable part of its boundary (grey regions). The extended domain is the unit square divided into 10055 finite elements (maximal element diameter $h=0.025$ ). The source $f$ is uniform inside the white circle and zero outside, $\int_{\Omega} f=1$. Computed with $\tau=0.05$ : middle — the final stationary pile surface $W_{\varepsilon}$; right—levels of the corresponding stationary surface flux, $\left|\underline{Q}_{\varepsilon}\right|$. 

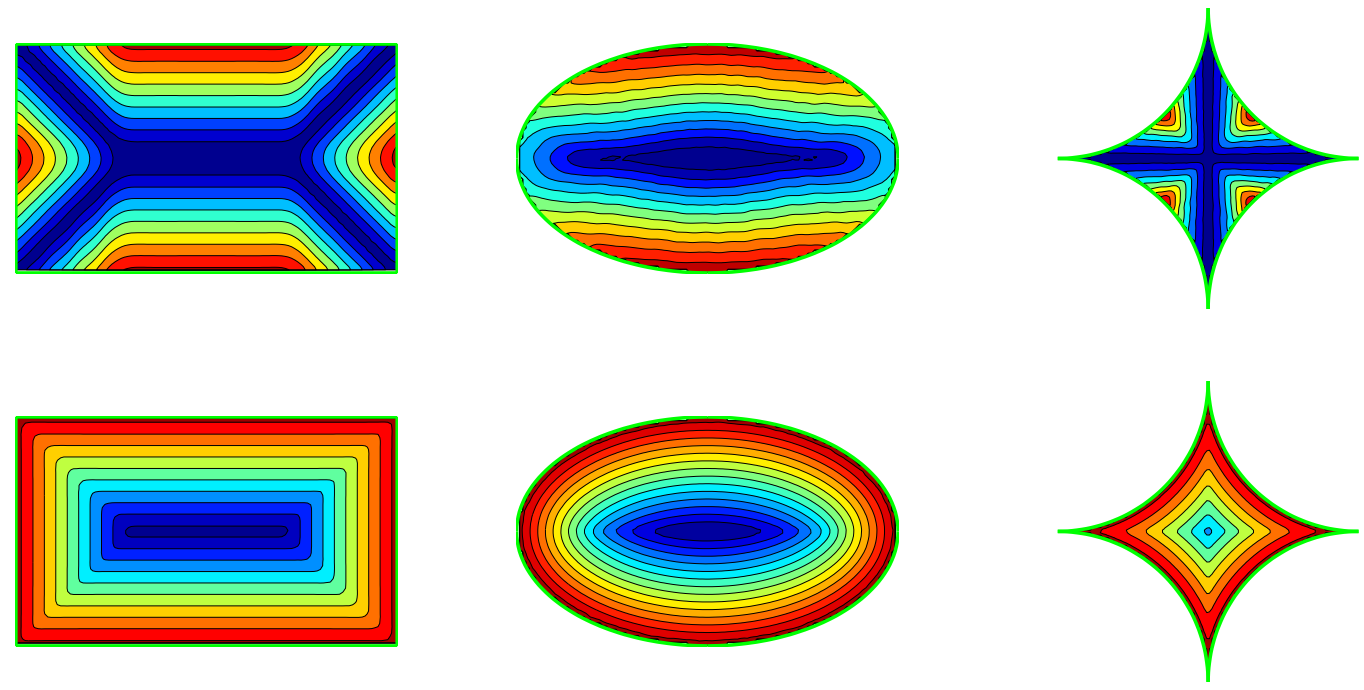

FIG. 6. Magnetization of homogeneous superconductive cylinders of different cross-sections. Upper row-the levels of the electric field $\left|\underline{E}_{\varepsilon}\right|$, lower row—the levels of the magnetic field $H_{\varepsilon}$ and the current contours (shown for $t=0.3$ ).

total domain $\Omega$ is the unit square, and the source is constant in time and uniform inside its support (the white circle). The final pile shape (Fig. 5 middle) is shown jointly with the corresponding surface flux (right).

As was noted above, the simple transformation $R: \underline{e}=\left(e_{1}, e_{2}\right)^{T} \mapsto\left(e_{2},-e_{1}\right)^{T}$ enables us to use the same Raviart-Thomas element as in the sandpile problem for the superconductivity problem. The parameters in the numerical simulations below were chosen on assuming that the dimensionless variables $(\underline{x}, t, \ldots)$ were obtained from the original variables $\left(\underline{x}^{\prime}, t^{\prime}, \ldots\right)$ as follows:

$$
\underline{x}=\frac{\underline{x}^{\prime}}{L}, \quad t=\frac{t^{\prime}}{t_{0}}, \quad \underline{j}=\frac{j^{\prime}}{j_{0}}, \quad h=\frac{h^{\prime}}{L j_{0}}, \quad \underline{e}=\frac{\underline{e}^{\prime} t_{0}}{L^{2} j_{0}},
$$

where $L$ is the characteristic cross-section size (the maximal horizontal extension in the plots below), and $j_{0}$ is the critical current density $j_{c}$ or its maximal value if the sample is not homogeneous. For superconductivity problems the source $f=-\mathrm{d} h_{e}(t) / \mathrm{d} t$ does not depend on $\underline{x}$. In the examples below we assumed that $f<0$ is constant and $t_{0}$ is chosen to make $f=-1$.

If the superconductor is homogeneous, i.e. $j_{c}$ does not depend on $\underline{x}$, and the domain $\Omega$ is simply connected, the magnetic field $h$ can be found analytically (see, e.g., [4]). For a growing external field $h_{e}(t)=t$ and $h^{0}(\underline{x})=0$, as in our next example, we get $h(\underline{x}, t)=-\min (\operatorname{dist}(\underline{x}, \partial \Omega), t)$. In Fig. 6, the electric and magnetic field approximations $\underline{E}_{\varepsilon}$ and $H_{\varepsilon}$, were computed numerically from the approximations of (1.16) and (1.17), respectively, for three different cross-sections. We note that the algorithm worked well also in the case of a non-Lipschitz domain with cusps. Even results obtained on a crude mesh were reasonably accurate. For the rectangular cross-section, using a regular $80 \times 48$ grid and $\tau=0.0125$, we obtained the magnetic field with an error in $L^{\infty}\left(\Omega_{T}\right)$ not exceeding $1 \%$. For full penetration, the electric field in superconductors with a rectangular crosssection has been found analytically in [5]; comparison showed that the electric field computed on 
such a mesh has an error in $L^{\infty}(\Omega)$ of about $2 \%$. In this case, also for the electric field we observed first order convergence in both $h$ and $\tau$ in the $L^{\infty}(\Omega)$ norm.

The next example shows magnetization of a non-homogeneous superconductor (the configuration is shown in Fig. 7). Clearly, the easy pathways of magnetic field penetration into the

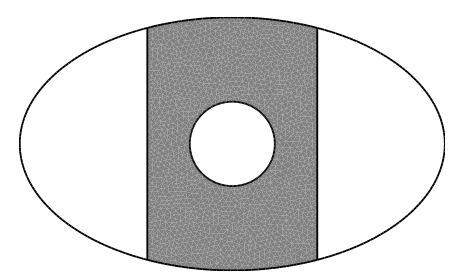

FIG. 7. Inhomogeneous superconductor. Grey region: $j_{c}=1$, white regions: $j_{c}=1 / 3$. Triangulation used: 7120 elements with the maximal diameter 0.02 .

superconductor go through the regions of lower critical current density; the electric field is strongest along these pathways (Fig. 8).
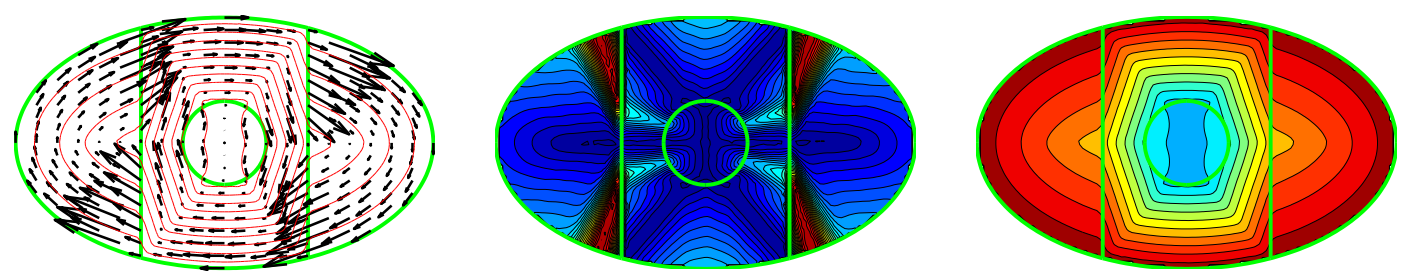

FIG. 8. Magnetization of inhomogeneous superconductor. Shown for $t=0.3$ : left-the electric field $\underline{E}_{\varepsilon}$ and the current contours; middle - the levels of $\left|\underline{E}_{\varepsilon}\right|$; right—-the levels of the magnetic field $H_{\varepsilon}$.

To model magnetization of a superconductor with a multiply connected cross-section, one can "fill" the holes and set $j_{c}=0$ there. It is, however, well known that eddy-current problems determine the electric field only inside conductors: the field in isolators remains non-unique (unlike the magnetic field, which is determined everywhere). Indeed, the stationary charges in isolators cannot influence the induced currents and, hence, the electric field in the conductors; the field in the isolators depends on the distribution of these charges. Our theoretical analysis also holds only for positive values of $j_{c}$. To deal with this complication it is possible to define a very small critical current density $j_{c}=\delta>0$ in the holes; our simulations show that the electric field in a superconductor is not sensitive to the value of $\delta$. In our last example (see Fig. 9), we set $\delta=10^{-7}$ for the circular hole and used 5394 elements with the maximal diameter 0.035 . As the external field starts to grow, the magnetic field penetrates the superconductor from its boundary, at first as if there were no hole in the cross-section. However, when the zone of penetration reaches the hole boundary, the magnetic field begins to penetrate the hole via an infinitely thin channel, similar to the runway in Fig. 1, and the electric field becomes singular. We note that the appearance of such a channel had been predicted by Badía-Majós and López [2], but they were unable to simulate it using their numerical method. 

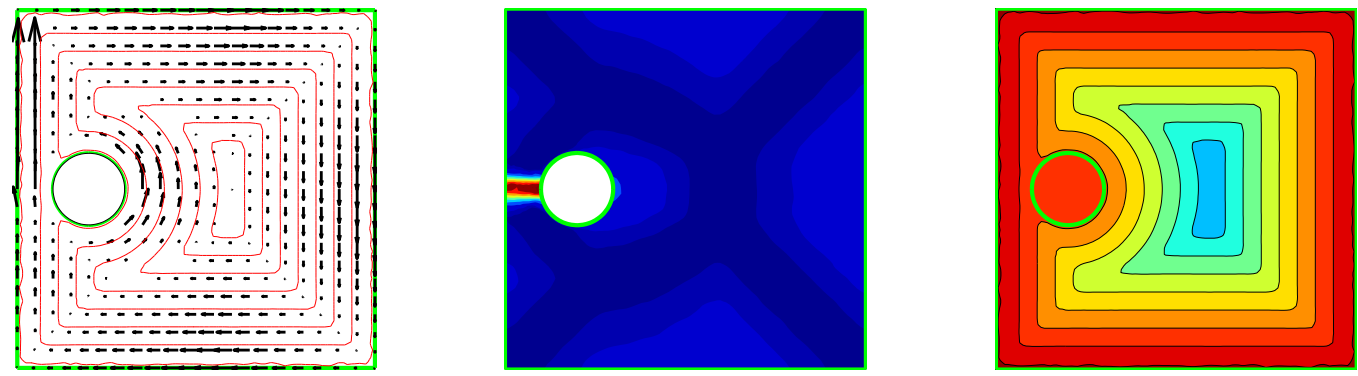

FIG. 9. Cylindrical superconductor with a hole. Shown for $t=0.6$ : left-the electric field $E_{\varepsilon}$ and the current contours, middle—the level contours of $\left|\underline{E}_{\varepsilon}\right|$, right—-the levels of $H_{\varepsilon}$. Note the "runway" (red region in the $\left|\underline{E}_{\varepsilon}\right|$ level plot, see pdf file) through which the magnetic field penetrates the hole and where the electric field is the strongest.

\section{Acknowledgements}

JWB acknowledges support from an Isaiah Berlin Israel Travel Award and The Blaustein Center for Scientific Cooperation.

\section{REFERENCES}

1. Aronson, G., Evans, L. C., \& Wu, Y. Fast/slow diffusion and growing sandpiles. J. Differential Equations 131 (1996), 304-335.

2. BAdíA-MAJós, A., \& LÓPEZ, C. Electric field in hard superconductors with arbitrary cross section and general critical current law. J. Appl. Phys. 95 (2004), 8035-8040.

3. Bahriawati, C., \& Carstensen, C. Three Matlab implementations of the lowest-order RaviartThomas MFEM with a posteriori error control. Comput. Methods Appl. Math. 5 (2005), 333-361. Zbl pre02243764 MR 2194203

4. BARrett, J. W., \& Prigozhin, L. Bean's model as the $p \rightarrow \infty$ limit of an evolutionary p-Laplacian equation. Nonlinear Anal. 42 (2000), 977-993. Zbl pre01526170 MR 1780448

5. Brandt, E. H. Electric field in superconductors with rectangular cross section. Phys. Rev. B 52 (1995), 15442-15457.

6. Cannarsa, P., \& CARdaliaguet, P. Representation of equilibrium solutions to the table problem for growing sandpiles. J. Eur. Math. Soc. 6 (2004), 435-464. Zbl 1084.35015 MR 2094399

7. Evans, L. C. Weak Convergence Methods for Nonlinear Partial Differential Equations. CBMS Reg. Conf. Ser. Math. 74, Amer. Math. Soc. Providence, RI (1990). MR 1034481

8. Feldman, M. Growth of a sandpile around an obstacle. Monge Ampère Equation: Applications to Geometry and Optimization, L. A. Caffarelli and M. Milman (eds.), Contemp. Math. 226, Amer. Math. Soc. Providence, RI (1999), 55-78. Zbl 0924.35176 MR 1660742

9. Folland, G. B. Real Analysis: Modern Techniques and their Applications. 2nd ed., Wiley-Interscience, New York (1984). Zbl 0549.28001 MR 0767633

10. Han, W., \& RedDy, B. D. Plasticity: Mathematical Theory and Numerical Analysis. Springer, New York (1999). Zbl 0926.74001 MR 1681061

11. Partial Differential Equation Toolbox User's Guide. The MathWorks (1995).

12. Prigozhin, L. Sandpiles and river networks: extended systems with nonlocal interactions. Phys. Rev. E 49 (1994), 1161-1167. MR 1379784 
13. Prigozhin, L. Variational model for sandpile growth. Europ. J. Appl. Math. 7 (1996), 225-235. Zbl 0913.73079 MR 1401168

14. Prigozhin, L. Variational inequalities in critical-state problems. Phys. D 167 (2004), 197-210. Zbl 1076.82034 MR 2093571

15. Prigozhin, L. Solutions to Monge-Kantorovich equations as stationary points of a dynamical system. arXiv:math.OC/0507330, http://xxx.tau.ac.il/abs/math.OC/0507330 (2005).

16. Roberts, J. E., \& Thomas, J.-M. Mixed and hybrid methods. Handbook of Numerical Analysis, Vol. II, P. G. Ciarlet and J.-L. Lions (eds.), North-Holland, Amsterdam (1991), 523-639. Zbl 0875.65090 MR 1115239

17. Temam, R. Mathematical Methods in Plasticity. Gauthier-Villars, Paris (1985). 